\title{
EXPLORING ANTICORRELATIONS AND LIGHT ELEMENT VARIATIONS IN NORTHERN GLOBULAR CLUSTERS OBSERVED BY THE APOGEE SURVEY
}

\author{
Szabolcs Mészáros ${ }^{1,2}$, Sarah L. Martell ${ }^{3}$, Matthew Shetrone ${ }^{4}$, Sara Lucatello ${ }^{5}$, Nicholas W. Troup ${ }^{6}$, Jo Bovy ${ }^{7}$, \\ Katia Cunha ${ }^{8,9}$, Domingo A. García-Hernández ${ }^{10,11}$, Jamie C. Overbeek ${ }^{2}$, Carlos Allende Prieto ${ }^{10,11}$, \\ Timothy C. Beers ${ }^{12}$, Peter M. Frinchaboy ${ }^{13}$, Ana E. García Pérez ${ }^{6,10,11}$, Fred R. Hearty ${ }^{14}$, Jon Holtzman ${ }^{15}$, \\ Steven R. Majewski ${ }^{6}$, David L. Nidever ${ }^{16}$, Ricardo P. Schiavon ${ }^{17}$, Donald P. Schneider ${ }^{14,18}$, Jennifer S. Sobeck ${ }^{6}$, \\ Verne V. Smith ${ }^{19}$, Olga Zamora ${ }^{10,11}$, and Gail Zasowski ${ }^{20}$ \\ ${ }^{1}$ ELTE Gothard Astrophysical Observatory, H-9704 Szombathely, Szent Imre Herceg st. 112, Hungary \\ ${ }^{2}$ Department of Astronomy, Indiana University, Bloomington, IN 47405, USA \\ ${ }^{3}$ Department of Astrophysics, School of Physics, University of New South Wales, Sydney, NSW 2052, Australia \\ ${ }^{4}$ University of Texas at Austin, McDonald Observatory, Fort Davis, TX 79734, USA \\ ${ }^{5}$ INAF-Osservatorio Astronomico di Padova, vicolo dell Osservatorio 5, I-35122 Padova, Italy \\ ${ }^{6}$ Department of Astronomy, University of Virginia, Charlottesville, VA 22904-4325, USA \\ ${ }^{7}$ Institute for Advanced Study, Einstein Drive, Princeton, NJ 08540, USA \\ ${ }^{8}$ University of Arizona, Tucson, AZ 85719, USA \\ ${ }^{9}$ Observatório Nacional, São Cristóvão, Rio de Janeiro, Brazil
${ }^{10}$ Instituto de Astrofísica de Canarias (IAC), E-38200 La Laguna, Tenerife, Spain \\ ${ }^{11}$ Universidad de La Laguna, Departamento de Astrofísica, E-38206 La Laguna, Tenerife, Spain \\ ${ }^{12}$ Department of Physics and JINA Center for the Evolution of the Elements, University of Notre Dame, Notre Dame, IN 46556, USA \\ ${ }^{13}$ Texas Christian University, Fort Worth, TX 76129, USA \\ ${ }^{14}$ Department of Astronomy and Astrophysics, The Pennsylvania State University, University Park, PA 16802, USA \\ ${ }^{15}$ New Mexico State University, Las Cruces, NM 88003, USA \\ ${ }^{16}$ Department of Astronomy, University of Michigan, Ann Arbor, MI 48109, USA \\ ${ }^{17}$ Astrophysics Research Institute, IC2, Liverpool Science Park, Liverpool John Moores University, 146 Brownlow Hill, Liverpool, L3 5RF, UK \\ ${ }^{18}$ Institute for Gravitation and the Cosmos, The Pennsylvania State University, University Park, PA 16802, USA \\ ${ }^{19}$ National Optical Astronomy Observatory, Tucson, AZ 85719, USA \\ ${ }^{20}$ Johns Hopkins University, Baltimore, MD 21218, USA \\ Received 2014 November 5; accepted 2015 January 20; published 2015 April 14
}

\begin{abstract}
We investigate the light-element behavior of red giant stars in northern globular clusters (GCs) observed by the SDSS-III Apache Point Observatory Galactic Evolution Experiment. We derive abundances of 9 elements (Fe, C, $\mathrm{N}, \mathrm{O}, \mathrm{Mg}, \mathrm{Al}, \mathrm{Si}, \mathrm{Ca}$, and $\mathrm{Ti}$ ) for 428 red giant stars in $10 \mathrm{GCs}$. The intrinsic abundance range relative to measurement errors is examined, and the well-known $\mathrm{C}-\mathrm{N}$ and $\mathrm{Mg}-\mathrm{Al}$ anticorrelations are explored using an extreme-deconvolution code for the first time in a consistent way. We find that $\mathrm{Mg}$ and $\mathrm{Al}$ drive the population membership in most clusters, except in M107 and M71, the two most metal-rich clusters in our study, where the grouping is most sensitive to $\mathrm{N}$. We also find a diversity in the abundance distributions, with some clusters exhibiting clear abundance bimodalities (for example M3 and M53) while others show extended distributions. The spread of $\mathrm{Al}$ abundances increases significantly as cluster average metallicity decreases as previously found by other works, which we take as evidence that low metallicity, intermediate mass AGB polluters were more common in the more metal-poor clusters. The statistically significant correlation of $[\mathrm{Al} / \mathrm{Fe}]$ with $[\mathrm{Si} / \mathrm{Fe}]$ in $\mathrm{M} 15$ suggests that ${ }^{28} \mathrm{Si}$ leakage has occurred in this cluster. We also present $\mathrm{C}, \mathrm{N}$, and $\mathrm{O}$ abundances for stars cooler than $4500 \mathrm{~K}$ and examine the behavior of $\mathrm{A}(\mathrm{C}+\mathrm{N}+\mathrm{O})$ in each cluster as a function of temperature and $[\mathrm{Al} / \mathrm{Fe}]$. The scatter of $\mathrm{A}(\mathrm{C}+\mathrm{N}$ $+\mathrm{O})$ is close to its estimated uncertainty in all clusters and independent of stellar temperature. $\mathrm{A}(\mathrm{C}+\mathrm{N}+\mathrm{O})$ exhibits small correlations and anticorrelations with $[\mathrm{Al} / \mathrm{Fe}]$ in $\mathrm{M} 3$ and $\mathrm{M} 13$, but we cannot be certain about these relations given the size of our abundance uncertainties. Star-to-star variations of $\alpha$-element $(\mathrm{Si}, \mathrm{Ca}, \mathrm{Ti})$ abundances are comparable to our estimated errors in all clusters.
\end{abstract}

Key words: stars: abundances - stars: AGB and post-AGB - stars: chemically peculiar - stars: evolution

Supporting material: machine-readable and VO tables

\section{INTRODUCTION}

Over the last two decades, the long-lasting idea of globular clusters (GCs) hosting single, simple stellar populations has changed dramatically. The classical paradigm of GCs being an excellent example of a simple stellar population, defined as a coeval and initially chemically homogeneous assembly of stars, has been challenged by observational evidence. The presence of chemical inhomogeneities, in most cases limited to the light elements (the chemical pairs $\mathrm{C}-\mathrm{N}, \mathrm{O}-\mathrm{Na}$, and $\mathrm{Mg}-\mathrm{Al}$ anticorrelated with each other), have been known for decades and recognized to be the signature of high-temperature H-burning. This was initially framed within a stellar evolutionary scenario (see, e.g., Kraft 1994, and references therein) given that GC abundance work based on high-quality data was limited to bright, evolved giants. It was only at the turn of the century that the availability of high-resolution spectrographs mounted on $8 \mathrm{~m}$ class telescopes made it possible to carry out studies on the compositions of stars down to the main-sequence, which revealed light element variations analogous to those found among giants (Briley et al. 1996; Gratton et al. 2001; Ramírez $\&$ Cohen 2002). Given that the atmospheres of warm main- 
sequence stars in large part retain the composition of the gas from which they were formed, the unavoidable conclusion was that the abundance inhomogeneities are of primordial origin.

The most extensive spectroscopic survey of GCs undertaken so far (Carretta et al. 2009a, 2009b, 2009c) revealed that these inhomogeneities are ubiquitous in Galactic GCs, though they do not appear to occur in other star formation environments. However, the extent of the inhomogeneity varies from cluster to cluster, and appears to correlate strongly with the presentday total mass of the GCs, and also with metallicity. The improvement in available instrumentation and techniques has also led to the discovery of a much higher degree of complexity of GC color-magnitude diagrams. In fact, while some clusters seem to photometrically comply with the simple stellar population paradigm, a growing number of them are found to be characterized by multiple main sequences and/or subgiant and/or giant branches (Piotto et al. 2007; Milone et al. 2008, e.g.,), which have been associated with variations in the content of $\mathrm{He}$ and $\mathrm{CNO}$, as well as age spread (DAntona et al. 2005; Cassisi et al. 2008).

This observational evidence led to a general scenario where GCs host multiple stellar populations. These are often assumed to be associated with different stellar generations: the ejecta of the slightly older stars, probably mixed with varying amounts of gas from the original star-forming cloud, creates a subsequent younger generation of stars (see e.g., Decressin et al. 2007; D'Ercole et al. 2008), though alternative scenarios are also being considered (see, e.g., Bastian et al. 2013). It is believed that only a fraction of the first generation of stars can contribute to the internal enrichment. The difference in ages among the stellar generations is actually relatively small for the majority of the clusters (with a few exceptions such as, e.g., $\omega$ Cen or M22) and is confined to a couple of hundreds of Myr.

The details of this formation scenario are still far from being understood. The origin of the polluting material remains to be established and it has obvious bearings on the timescales for the formation of the cluster itself and its mass budget. The observed wide star-to-star variations in $\mathrm{C}, \mathrm{N}, \mathrm{O}, \mathrm{Na}$, and $\mathrm{Al}$ found in each Galactic GC, coupled with the uniformity in $\mathrm{Fe}$ and $\mathrm{Ca}$ (apart from a few notable exceptions), provide quite stringent constraints and argue against anything but a minor contribution from supernovae (Carretta et al. 2009a). Proposed candidate polluters include intermediate mass stars in their asymptotic giant branch (AGB) phase (Ventura et al. 2001), fast rotating massive stars losing mass during their main sequence phase (Decressin et al. 2007), novae (Maccarone \& Zurek 2012), and massive binaries (de Mink et al. 2009). These potential contributions obviously operate on different timescales and require a different amount of stellar mass in the first generation. All of the candidates proposed so far fall short of reproducing the full variety of observations. Advances in the theoretical modeling of star formation and evolution are likely needed to improve our understanding of these issues, including the spanning of a larger range of the parameter space (e.g., mass, metallicity, and mass loss). However, from an observational point of view, the increase in the high-quality abundance work available for GCs, both in the sheer number of stars and clusters, as well as in terms of chemical species considered, is paramount, as it creates a more complete picture of the phenomena involved.

The Apache Point Observatory Galactic Evolution Experiment (APOGEE; S.R. Majewski et al. 2015, in preparation) is a three-year, near-infrared (15090-16990 A; Wilson et al. 2012), high-resolution spectroscopic survey of about 100,000 red giant stars included as part of the third Sloan Digital Sky Survey (SDSS-III Eisenstein et al. 2011). With a nominal resolving power of 22,500, APOGEE is deriving abundances of up to 15 elements for nearly 100,000 stars, although fewer elements are generally detected in weak-lined metal-poor stars. APOGEE is in a unique position among the various Galactic spectroscopic surveys such as Gaia-ESO, (Gilmore et al. 2012), RAVE (Steinmetz et al. 2012), and GALAH (Freeman 2012), as it uses the Sloan $2.5 \mathrm{~m}$ telescope at Apache Point Observatory (Gunn et al. 2006), and thereby has access to the northern hemisphere. APOGEE observes a large sample of northern GCs, something that makes it possible to analyze these clusters in a homogeneous way, which has not been done before for these objects.

The study of GCs with APOGEE plays an important role not just because it has access to many northern GCs. Its highresolution near-IR spectra allow the simultaneous determination of many elemental abundances generally not available in optical spectroscopic work of GC stars. $\mathrm{C}$ and $\mathrm{N}$, which are elements heavily affected by the pollution phenomenon in GCs, are often not included in studies of metal-poor stars because the strongest features $(\mathrm{CH}$ and $\mathrm{CN})$ lie in the near-UV, far from the optical lines of $\mathrm{Na}, \mathrm{Mg}$, and $\mathrm{Al}$, and thus multiple detectors or setups are required to obtain both sets. In addition, because these studies usually focus on fairly red stars, longer exposure times are required to acquire sufficient supernova remnants to analyze the near-UV features.

The spectra used in this paper are publicly available as part of the 10th data release (DR10, Ahn et al. 2014) of SDSS-III. The initial set of stars selected were the same used by Mészáros et al. (2013) to check the accuracy and precision of APOGEE parameters published in DR10. However, instead of using the automatic ASPCAP pipeline, we will make use of photometry and theoretical isochrones to constrain the effective temperature $\left(T_{\text {eff }}\right)$ and surface gravity $\log g$ and use an independent semi-automated method for elemental abundance determination for 10 northern GCs. Some of these clusters are well studied, such as M3, M13, M92, and M15, while others have been poorly studied (NGC 5466), or have been only recently discussed in the literature, such as M2 (Yong et al. 2014).

\section{ABUNDANCE ANALYSIS}

\subsection{Target Selection}

Table 1 lists the GCs APOGEE observed in its first year, along with the adopted $[\mathrm{Fe} / \mathrm{H}], E(B-V)$, and ages from the literature. Targets were selected as cluster members if (1) there is published abundance information on the star as a cluster member, (2) the star is a radial velocity member, or (3) if it has a probability $>50 \%$ of being a cluster member based on their proper motion adopted from the literature. After this initial selection, we checked the position of stars in the $T_{\text {eff }}-\log g$ diagram based on APOGEE observations and deleted those that were not red giant branch (RGB) stars. Stars that have metallicity 0.3 dex (typically $3 \sigma$ scatter) larger or smaller than the cluster average also need to be deleted, but this last step resulted in no rejections. The cluster target selection process is described in more detail in Zasowski et al. (2013). The final sample consists of 428 stars from 10 GCs. High signal-to-noise ratio $(\mathrm{S} / \mathrm{N})$ spectra are essential to determine abundances from 
Table 1

Properties of Clusters from the Literature

\begin{tabular}{llrrc}
\hline \hline ID & Name & $N^{\mathrm{a}}$ & {$[\mathrm{Fe} / \mathrm{H}]^{\mathrm{b}}$} & $E(B-V)$ Ref. $^{\mathrm{a}}$ \\
\hline NGC 7078 & M15 & 23 & $-2.37 \pm 0.02$ & 0.10 \\
NGC 6341 & M92 & 47 & $-2.31 \pm 0.05$ & 0.02 \\
NGC 5024 & M53 & 16 & $-2.10 \pm 0.09$ & 0.02 \\
NGC 5466 & & 8 & $-1.98 \pm 0.09$ & 0.00 \\
NGC 6205 & M13 & 81 & $-1.53 \pm 0.04$ & 0.02 \\
NGC 7089 & M2 & 18 & $-1.65 \pm 0.07$ & 0.06 \\
NGC 5272 & M3 & 59 & $-1.50 \pm 0.05$ & 0.01 \\
NGC 5904 & M5 & 122 & $-1.29 \pm 0.02$ & 0.03 \\
NGC 6171 & M107 & 42 & $-1.02 \pm 0.02$ & 0.33 \\
NGC 6838 & M71 & 12 & $-0.78 \pm 0.02$ & 0.25 \\
\hline
\end{tabular}

${ }^{\mathrm{a}} N$ is the number of stars observed in each cluster.

b $[\mathrm{Fe} / \mathrm{H}]$ references: Harris $(1996,2010$ edition) clusters are listed in order of the average cluster metallicity determined in this paper.

${ }^{\mathrm{c}} E(B-V)$ references: (1) Harris (1996, 2010 edition).

atomic and molecular features, thus all selected targets have at least $\mathrm{S} / \mathrm{N}=70$ as determined by Mészáros et al. (2013).

\subsection{Atmospheric Parameters}

Abundances presented in this paper are defined for each individual element $\mathrm{X}$ heavier than helium as

$$
[\mathrm{X} / \mathrm{H}]=\log _{10}\left(n_{\mathrm{X} / n_{\mathrm{H}}}\right)_{\mathrm{star}}-\log _{10}\left(n_{\mathrm{X}} / n_{\mathrm{H}}\right)_{\odot}
$$

where $n_{\mathrm{X}}$ and $n_{\mathrm{H}}$ are respectively the number of atoms of element $\mathrm{X}$ and hydrogen, per unit volume in the stellar photosphere.

To derive abundances from stellar spectra, we first have to estimate four main atmospheric parameters: $T_{\text {eff }}, \log g$, microturbulent velocity, and overall metallicity $([\mathrm{Fe} / \mathrm{H}])$. In the following sub-sections we present our methodology for determining these parameters and our reasons for not using the values available for each star from the APOGEE Stellar Parameters and Chemical Abundances Pipeline (ASPCAP; García Pérez et al. 2015, in preparation) in DR10. In order to evaluate the accuracy and precision of the ASPCAP parameters, Mészáros et al. (2013) carried out a careful comparison with literature values using 559 stars in 20 open and GCs.

These clusters were chosen to cover most of the parameter range of stars APOGEE is expected to observe. Mészáros et al. (2013) provided a detailed explanation of the accuracy and precision of these parameters, and also derived empirical calibrations for $T_{\text {eff }}, \log g$, and $[\mathrm{M} / \mathrm{H}]$ using literature data. In the sections below we will briefly review these calibrations along with their limitations.

\subsection{The Effective Temperature}

We adopt a photometric effective temperatures calculated from the $J-K_{s}$ colors using the equations of González Hernández \& Bonifacio (2009). Their calibration was chosen because of its proximity of only $30-40 \mathrm{~K}$ to the absolute temperature scale. De-reddened $J-K_{s}$ were calculated the same way as by Mészáros et al. (2013), from $E(B-V)$, listed in Table 1 for each cluster, using $E\left(J-K_{s}\right)=0.46 \cdot E(B-V)$.

The ASPCAP DR10 effective temperatures were compared with photometric ones using calibrations by González Hernández \& Bonifacio (2009) based on 2MASS $J-K_{s}$ colors (Strutskie et al. 2006). Mészáros et al. (2013) found that small systematic differences, in the range of 100-200 K, are present between the ASPCAP and photometric temperatures. The ASPCAP DR10 $T_{\text {eff }}$ were also compared to literature spectroscopic temperatures, and the average of these differences were found to be negligible. The corrected ASPCAP DR10 temperatures were calculated between 3500 and $5500 \mathrm{~K}$ using a calibration relation derived from the comparison with the González Hernández \& Bonifacio (2009) scale. ASPCAP DR10 raw temperatures above $5000 \mathrm{~K}$ in metal-poor stars showed significant, 300-500 K offsets compared to photometry, and are thus believed to be not accurate enough for abundance analysis. This issue is mostly limited to metal-poor stars, and does not affect stars at $[\mathrm{Fe} / \mathrm{H}]>-1$, where the vast majority of APOGEE targets are.

The adoption of a purely photometric temperature scale enables us to be somewhat independent of ASPCAP (while still using the same spectra), which gives important comparison data for future ASPCAP validation. Besides providing an independent comparison data set for APOGEE, the photometric temperatures allowed us to include stars in the sample that are hotter than $5000 \mathrm{~K}$. Because of these reasons, the final results presented in this paper are based on the photometric temperatures, and we only use the ASPCAP DR10 raw temperatures to estimate our errors related to the atmospheric parameters.

\subsection{The Metallicity}

The APOGEE DR10 release contains metallicities derived by ASPCAP for all stars analyzed, thus providing an alternative scale to manually derived metallicities. That metallicity, $[\mathrm{M} / \mathrm{H}]$, tracks all metals relative to the Sun, and gives the overall metallicity of the stars because it was derived by fitting the entire wavelength region covered by the APOGEE spectrograph. This is different from most literature publications that use Fe lines to track metallicity in a stellar atmosphere. We use $[\mathrm{Fe} / \mathrm{H}]$ in this paper whenever we refer to metallicity presented here, because we use $\mathrm{Fe}_{\mathrm{I}}$ lines to measure it. For the most part, one can treat values of $[\mathrm{M} / \mathrm{H}]$ as if they were $[\mathrm{Fe} / \mathrm{H}]$. When ASPCAP metallicity was compared with individual values from high-resolution observations from the literature, a difference of $0.1 \mathrm{dex}$ is found below $[\mathrm{M} / \mathrm{H}]=-1$, and this discrepancy increased with decreasing metallicity reaching $0.2-0.3$ dex around $[\mathrm{M} / \mathrm{H}]=-2$ (Mészáros et al. 2013). The calibrated DR10 metallicities map well onto $[\mathrm{Fe} / \mathrm{H}]$, because the calibration process uses $[\mathrm{Fe} / \mathrm{H}]$ values from the optical.

Because an alternative metallicity based on only iron lines did not exist for APOGEE in DR10, and because of these small zero point offsets, we decided to derive our own $[\mathrm{Fe} / \mathrm{H}]$ based on $\mathrm{Fe}$ I lines found in the $\mathrm{H}$ band, instead of using the published APOGEE DR10 $[\mathrm{M} / \mathrm{H}]$ values. We assumed that all stars have the literature cluster mean metallicity before starting the calculations, after which small wavelength windows around the Fe lines listed in Table 3 were used to revise the individual star metallicities.

\subsection{The Surface Gravity}

In this study we adopt gravities from stellar evolution calculations. Following Mészáros et al. (2013), we derive gravities for our sample using isochrones from the Padova group (Bertelli et al. 2008, 2009). The cluster metallicities collected from the literature used in the isochrones are listed in 
Table 2

Properties of Stars Analyzed

\begin{tabular}{|c|c|c|c|c|c|c|c|c|c|c|c|c|c|}
\hline 2MASS ID & Cluster & $v_{\text {helio }}$ & $T_{\text {eff }}$ & $\log g$ & {$[\mathrm{Fe} / \mathrm{H}]$} & {$[\mathrm{C} / \mathrm{Fe}]$} & {$[\mathrm{N} / \mathrm{Fe}]$} & {$[\mathrm{O} / \mathrm{Fe}]$} & {$[\mathrm{Mg} / \mathrm{Fe}]$} & {$[\mathrm{Al} / \mathrm{Fe}]$} & {$[\mathrm{Si} / \mathrm{Fe}]$} & {$[\mathrm{Ca} / \mathrm{Fe}]$} & {$[\mathrm{Ti} / \mathrm{Fe}]$} \\
\hline $2 \mathrm{M} 21301565+1208229$ & M15 & -104.5 & 4836 & 1.56 & -2.12 & $\ldots$ & $\ldots$ & $\ldots$ & 0.16 & -0.06 & 0.35 & 0.19 & $\ldots$ \\
\hline $2 \mathrm{M} 21301606+1213342$ & M15 & -108.3 & 4870 & 1.64 & -2.31 & $\ldots$ & $\ldots$ & $\ldots$ & 0.10 & 0.57 & 0.46 & 0.53 & $\ldots$ \\
\hline $2 \mathrm{M} 21304412+1211226$ & M15 & -102.7 & 4715 & 1.28 & -2.12 & $\ldots$ & $\ldots$ & $\ldots$ & -0.45 & 0.63 & 0.60 & 0.35 & $\ldots$ \\
\hline $2 M 21290843+1209118$ & M15 & -106.0 & 4607 & 1.03 & -2.07 & $\ldots$ & $\ldots$ & $\ldots$ & -0.11 & 0.75 & 0.41 & $\ldots$ & $\ldots$ \\
\hline $2 \mathrm{M} 21294979+1211058$ & M15 & -107.6 & 4375 & 0.56 & -2.31 & -0.44 & 0.95 & 0.44 & 0.17 & 0.64 & 0.44 & 0.06 & $\ldots$ \\
\hline
\end{tabular}

(This table is available in its entirety in machine-readable and Virtual Observatory (VO) forms.)

Table 1, while the ages of all clusters were chosen to be $10 \mathrm{Gyr}$. The final set of temperatures and gravities corresponding to them from the isochrones are listed in Table 2.

The ASPCAP DR10 surface gravities were compared to both isochrones and Kepler (Borucki et al. 2010) asteroseismic targets observed by APOGEE (Pinsonneault et al. 2014) to estimate their accuracy and precision. An average difference of 0.3 dex was found at solar metallicity in both cases, but this increased to almost 1 dex for very metal-poor stars. The asteroseismic gravities are believed to have errors in the range of 0.01-0.05 dex, thus far superior to spectroscopic measurements, but they are only available for metal-rich stars with $[\mathrm{M} / \mathrm{H}]>-1.0$. The discrepancy between spectroscopic and asteroseismic surface gravities is a topic of ongoing investigation (e.g., Epstein et al. 2014; Pinsonneault et al. 2014). The final calibration from Mészáros et al. (2013) combined surface gravities derived from isochrones below $[\mathrm{M} / \mathrm{H}]<-1.0$ with the asteroseismic data set for high metallicities. Values after the calibration still show some small $(<0.1 \mathrm{dex})$ offsets and large scatter around the isochrones for the GCs. Thus, in order to minimize errors in abundances related to the uncertainty in the ASPCAP corrected spectroscopic gravities, we decided to use the pure isochrone gravities in this study.

\subsection{The Microturbulent Velocity}

A relation between microturbulent velocity and surface gravity, $v_{\text {micro }}=2.24-0.3 \times \log g$, was used for ASPCAP analyses in DR10 (Mészáros et al. 2013). For consistency with ASPCAP, and for easier future comparisons with APOGEE results, we adopted that equation in this work.

\section{AUTOSYNTH}

The program called autosynth was developed especially for this project to simplify the large amount of synthesis required for abundance determination. The program takes atmospheric parameters as input and carries out spectral synthesis to derive elemental abundances. The core of autosynth is MOOG2013 ${ }^{21}$ (Sneden 1973), which does the spectrum synthesis, while autosynth compares the synthetic spectrum with the observed spectrum and determines the best abundances with $\chi^{2}$ minimization in wavelength windows specified by the user. The program can read the MOOG formatted ATLAS and MARCS model atmospheres and can also convert the original ATLAS and MARCS formats into a MOOG compatible format.

The line list adopted for this study includes both atomic and molecular species. It is an updated version of what was used for the DR10 results, version m201312160900 (also used for

\footnotetext{
${ }^{21}$ http://as.utexas.edu/ chris/moog.html
}

DR12, APOGEE's next public data release; Alam et al. 2015; Holtzman et al. 2015), and includes atomic and molecular species. The molecular line list is a compilation of literature sources including transitions of $\mathrm{CO}, \mathrm{OH}, \mathrm{CN}, \mathrm{C}_{2}, \mathrm{H}_{2}$, and $\mathrm{SiH}$. All molecular data are adopted without change with the exception of a few obvious typographical corrections. The atomic line list was compiled from a number of literature sources and includes theoretical, astrophysical, and laboratory oscillator strength values. These literature line positions, oscillator strengths, and damping values were allowed to vary in order to fit to the solar spectrum and the spectrum of Arcturus, thus generating a tuned astrophysical line list. The solution is weighted such that the solar solution has twice the weight as the Arcturus solution to properly consider the fact that the abundance ratios in Arcturus are more poorly understood than those of the Sun. The code used for this process was based on the LTE spectral synthesis code MOOG (Sneden 1973) but adapted to our unique needs. For lines with laboratory oscillator strengths, we did not allow the astrophysical gf value to vary beyond twice the error quoted by the source. A more detailed description of this process and the line list can be found in M. Shetrone et al. (2015, in preparation).

The choice for the local continuum set can greatly affect the derived abundances, thus we needed a reliable automated way to determine the continuum placement. This was done with a separate $\chi^{2}$ minimization from the one that was used for the abundance determination. Continuum normalized observation points around 1 are multiplied by a factor between 0.7 and 1.1 with 0.001 steps for each synthesis emulating slightly different choices for the location of the local continuum. Multiplication is necessary because it preserves the original spectrum. The $\chi^{2}$ near the continuum is calculated and compared to the continuum of the observation and minimized separately for every abundance step. The $\chi^{2}$ calculation for the abundances determination happens between certain flux ranges (usually between 0.3 and 1.1) using the continuum placement determined in the previous step.

\subsection{Individual Abundances}

The individual abundances were determined using the 1D Local Thermodynamic Equilibrium (LTE) model atmospheres calculated with ATLAS9 (Kurucz 1979). The model atmospheres were generated using solar reference abundances from Asplund et al. (2005), the same way as the main APOGEE model atmosphere database was generated (Mészáros et al. 2012). Because the overall metallicity of these clusters were well known from the literature, initially we calculated atmospheres using the average literature metallicity for each cluster adopting the photometric effective temperatures and isochrone 
Table 3

Wavelength Regions

\begin{tabular}{|c|c|c|}
\hline Element & $\log (\mathrm{N})^{\mathrm{a}}$ & Wavelength $(\AA)^{\mathrm{b}}$ \\
\hline $\mathrm{Fe}$ & 7.45 & $\begin{array}{c}15210-15213.5 ; 15397-15401 ; 15651-15654 \\
15966-15973 ; 16044-16048 ; 16156-16160 \\
16168-16171\end{array}$ \\
\hline $\mathrm{C}$ & 8.39 & $\begin{array}{l}15572-15606 ; 15772-15791 ; 15980-16037 \\
16172-16248 ; 16617-16677 ; 16839-16870\end{array}$ \\
\hline $\mathrm{N}$ & 7.78 & $15240-15417$ \\
\hline $\mathrm{O}$ & 8.66 & $\begin{array}{l}15267-15272 ; 15281-15288 ; 15372-15380 \\
15386-15390 ; 15394-15397 ; 15404-15414 \\
15499-15502 ; 15508-15511 ; 15539-15542 \\
15561-15566 ; 15569-15574 ; 15887-15904 \\
16188-16198 ; 16207-16213 ; 16233-16237 \\
16244-16247 ; 16251-16261 ; 16300-16305 \\
16314-16319 ; 16707-16714 ; 16718-16720 \\
16731-16735 ; 16888-16892 ; 16898-16912\end{array}$ \\
\hline $\mathrm{Mg}$ & 7.53 & $15741-15757 ; 15767-15773$ \\
\hline $\mathrm{Al}$ & 6.37 & $16720-16727 ; 16751-16759 ; 16765-16770$ \\
\hline $\mathrm{Si}$ & 7.51 & $\begin{array}{l}15962-15966 ; 16062-16066 ; 16097-16101 \\
16218-16223 ; 16683-16687 ; 16830-16834\end{array}$ \\
\hline $\mathrm{Ca}$ & 6.31 & $16139-16143 ; 16153.5-16164$ \\
\hline $\mathrm{Ti}$ & 4.90 & $15546.5-15549.5 ; 15718-15721.5$ \\
\hline
\end{tabular}

${ }^{\mathrm{a}}$ The solar reference abundances are from Asplund et al. (2005).

${ }^{\mathrm{b}}$ Vacuum wavelength.

gravities. These initial model atmospheres were later revised to have consistency with the synthesis.

The windows used to derive the individual abundances were determined based on the analysis of FTS stars in the H-band using the APOGEE line list by Smith et al. (2013). In the case of $\mathrm{Fe}$ we measured $[\mathrm{Fe} / \mathrm{M}]$, relative to the literature cluster metallicity for each line. The abundance of $\mathrm{Na}$ is very important in discussing the spread of $\mathrm{O}$ in GCs, and two Na lines are available in the APOGEE spectral band. However, these two $\mathrm{Na}$ lines are weak even at solar metallicities. We carried out a number of tests attempting to derive $\mathrm{Na}$ abundances, but we found that the two Na lines become very weak around $[\mathrm{Fe} / \mathrm{H}]=-0.5$, and non-detectable below about -0.7 , thus we were not able to determine $\mathrm{Na}$ abundances for any of our targets. The list of wavelength regions used in our analysis and the solar reference values for each element are listed in Table 3. Figures 1 and 2 show examples of observed $\mathrm{Fe}, \mathrm{Mg}, \mathrm{Al}, \mathrm{OH}, \mathrm{CO}$, and $\mathrm{CN}$ line profiles and their fitted synthesis for one star from M71 and M13. The wavelength regions shown in these figures are only a fraction of what has been used from Table 3 .

$\mathrm{CN}$ lines spread over most of the $\mathrm{H}$ band, hence it is important to calculate the $\mathrm{CNO}$ abundances before the atomic ones. It is also important to use self-consistent model atmospheres because stars in GCs exhibit low carbon and high $\alpha$ content, which significantly alters the structure of the atmosphere compared to a solar scaled one (Mészáros et al. 2012). Taking into account all this, we developed the following procedure to produce the final abundances for each star:

1. A model atmosphere is generated using literature cluster average metallicities, the photometric temperature, and an isochrone gravity. Because all of our targets are RGB stars, we choose $[\mathrm{C} / \mathrm{Fe}]=-0.5,[\mathrm{O} / \mathrm{Fe}]=0.3$, and $[\mathrm{N} /$ $\mathrm{Fe}]=0.5 \mathrm{dex}$ for this initial model.
2. Individual $\mathrm{Fe}$ I lines are fit with autosynth, and an average $[\mathrm{Fe} / \mathrm{H}]$ is calculated for each star.

3. A new model atmosphere is calculated using this new $[\mathrm{Fe} / \mathrm{H}]$ value, but still using the starting $\mathrm{CNO}$ abundances.

4. We set the abundances of $\mathrm{C}, \mathrm{N}$, and $\mathrm{O}$ before the remaining elements, because they can have a significant effect on the atmospheric structure in cool stars. Since molecular features generally disappear from metal-poor spectra above $4500 \mathrm{~K}$, we divide our stars into two temperature groups. For the stars cooler than $4500 \mathrm{~K}$, we first determine $[\mathrm{O} / \mathrm{Fe}]$ using $\mathrm{OH}$ lines, then create a new model atmosphere with $[\alpha / \mathrm{Fe}]$ equal to $[\mathrm{O} / \mathrm{Fe}]$. We then determine $\mathrm{C}$ and $\mathrm{O}$ abundances from $\mathrm{CO}$ lines, then recreate the model atmosphere again with these new $[\mathrm{C} /$ $\mathrm{Fe}]$ and $[\mathrm{O} / \mathrm{Fe}]$ abundances. Finally, we derive $\mathrm{N}$ abundance using $\mathrm{CN}$ lines. For stars hotter than $4500 \mathrm{~K}$, we leave the $\mathrm{C}, \mathrm{N}$, and $\mathrm{O}$ abundances at their inital values.

5. The abundances of the remaining elements $(\mathrm{Mg}, \mathrm{Al}, \mathrm{Si}$, $\mathrm{Ca}$, and $\mathrm{Ti}$ ) are determined with autosynth, using the stellar parameters, metallicities, and $\mathrm{C}, \mathrm{N}$, and $\mathrm{O}$ abundances previously determined.

For each element, we average together the abundance results from the different wavelength regions to obtain final values. Although the size of each region is different, we did not find it necessary to use weights based on their ranges or line strengths, because that approach did not produce abundances significantly different from a straightforward average. Data reduction errors or missing data affected some of these regions, resulting in erroneous fits, and because of this we carefully examined each fit by eye. These wavelength regions were not included when constructing the final average abundances. The final abundance values are listed in Table 2 .

\subsection{Uncertainty Calculations}

\subsubsection{Systematic Uncertainties}

The uncertainty in the atmospheric parameters strongly affects the final abundances derived from some of the spectral features we consider. To test the sensitivity of abundances due to changes in the atmospheric parameters we used the results from the ASPCAP raw temperature scale. The same exact steps described in the previous section were followed, but instead of adopting the photometric temperature scale we adopt the ASPCAP DR10 raw temperature scale, which results in new surface gravities and microturbulent velocities. This way we could track systematics uncertainties sensitive to these parameters as well.

The differences in abundances as a function of photometric temperatures are demonstrated in Figure 3. The top left panel displays the differences in the measured abundances by using ASPCAP and photometric temperature, while the rest of the panels are assigned to each element. The color scale in all panels represents $\Delta T_{\text {eff. }}$. We defined the estimated errors associated with the atmospheric parameters based on the standard deviation around the mean differences between the two temperature scales. The calculated standard deviation of the difference in temperatures is $146 \mathrm{~K}$ (which we round to $150 \mathrm{~K}$ ). This standard deviation corresponds to the sum of the uncertainty in the photometric temperature and the ASPCAP temperature in quadrature. 

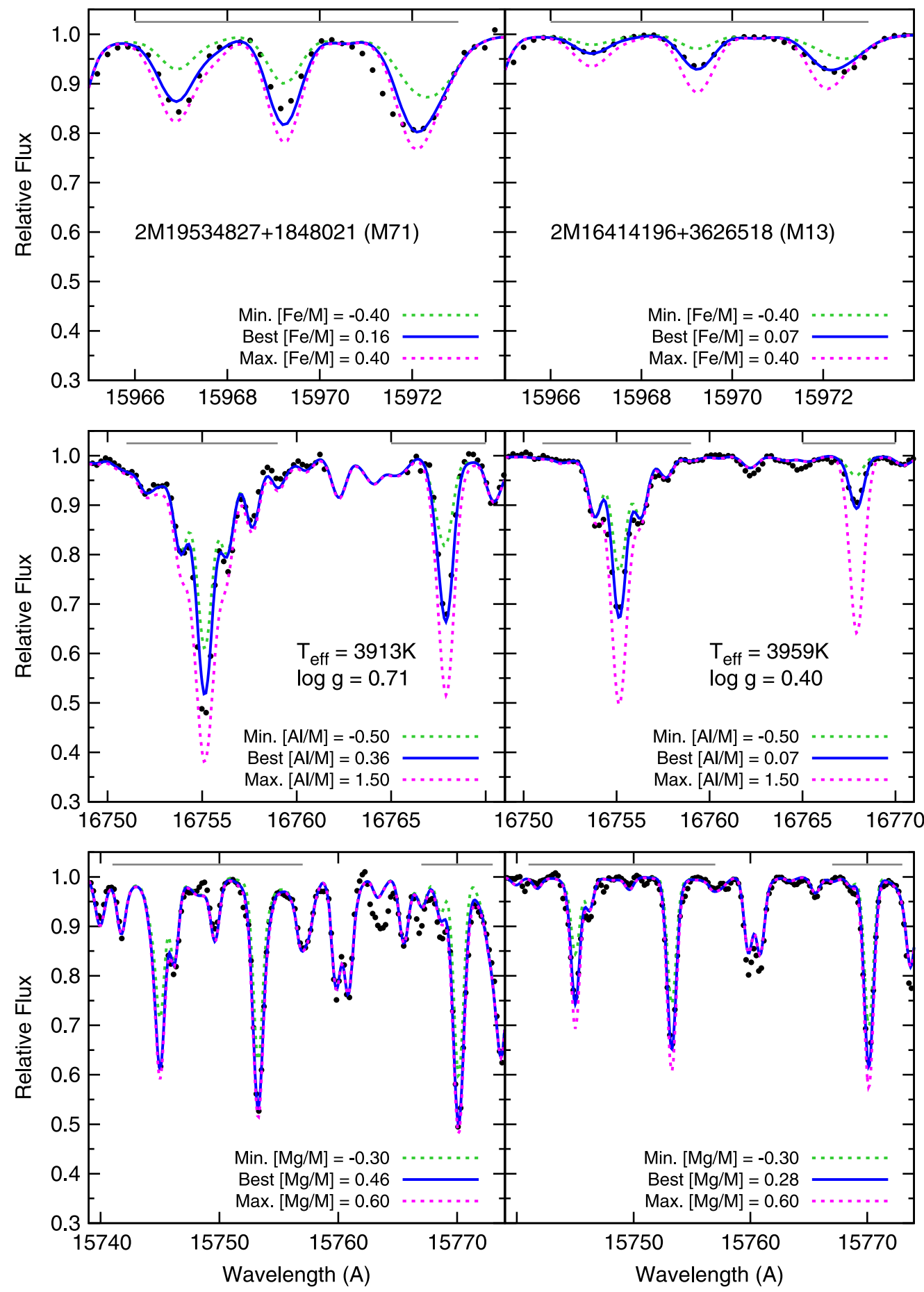

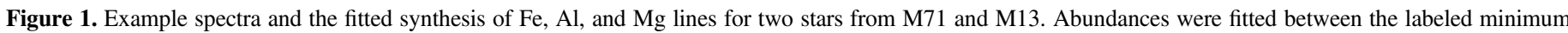

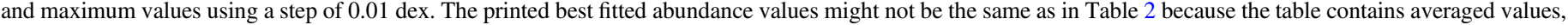
not individual fits.

In order to estimate the uncertainty of just the photometric temperature component, we carried out calculations of temperatures with varied $J-K_{s}$ colors, reddenings, and metallicities for M107. M107 was chosen because it is the cluster with the highest reddening in our sample. The uncertainty of 2MASS photometry is usually between 0.025 and $0.03 \mathrm{mag}$ for these stars, and by using 0.03 as a baseline, we estimate an uncertainty of 0.05 magnitude for the $J-K_{s}$ color. Changing $J-K_{s}$ by $0.05 \mathrm{mag}$ typically produces a change of $80 \mathrm{~K}$ in the photometric temperature. The reddening of M107 was changed by 0.03 , simulating a $10 \%$ uncertainty in reddening, and this produced a difference of about $40 \mathrm{~K}$ in the photometric temperature. A change of 0.1 dex in metallicity results in $1 \mathrm{~K}$ or less uncertainty in temperature; thus the uncertainties in metallicity can be neglected. By adding the uncertainty from photometry and reddening in quadrature, we estimate the uncertainty of the photometric temperature to be about $100 \mathrm{~K}$. The top panel of 

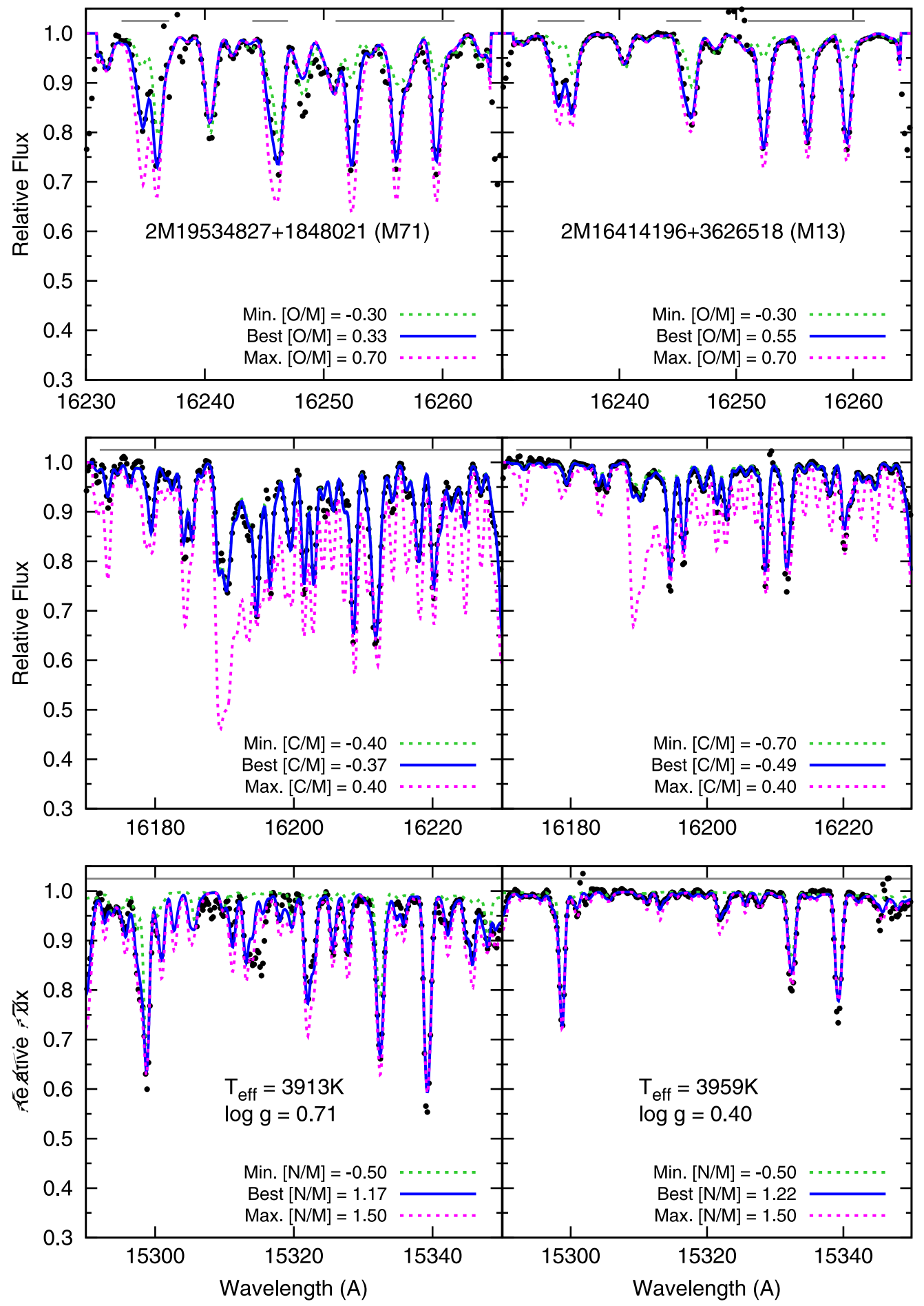

Figure 2. Example spectra and the fitted synthesis of $\mathrm{OH}, \mathrm{CO}$, and $\mathrm{CN}$ lines for two stars from M71 and M13. For more explanation see caption of Figure 1 and Section 3.1 .

Table 4 lists uncertainties associated with both 150 and $100 \mathrm{~K}$ changes in temperature.

In our methodology a $100 \mathrm{~K}$ change in temperature also introduces an 0.3 dex systematic difference in surface gravity, and $0.1 \mathrm{~km} \mathrm{~s}^{-1}$ in microturbulent velocity, so by coupling these two parameters to $T_{\text {eff }}$, our systematic uncertainties include the investigation of abundance sensitivity to these parameters as well.

\subsubsection{Internal Uncertainties}

Besides the uncertainty coming from the adopted atmospheric parameters, the uncertainty of the fit was also calculated using the $\sigma$ of the residuals between the best fit synthesis and the observation. These calculations estimate random uncertainties. This $\sigma$ of the fit is calculated within the windows used in the $\chi^{2}$ calculation. For determining the uncertainty of the fit, we multiply the observed spectra by $1+\sigma$ and $1-\sigma$ which simulates 
Table 4

Estimated Abundance Uncertainties

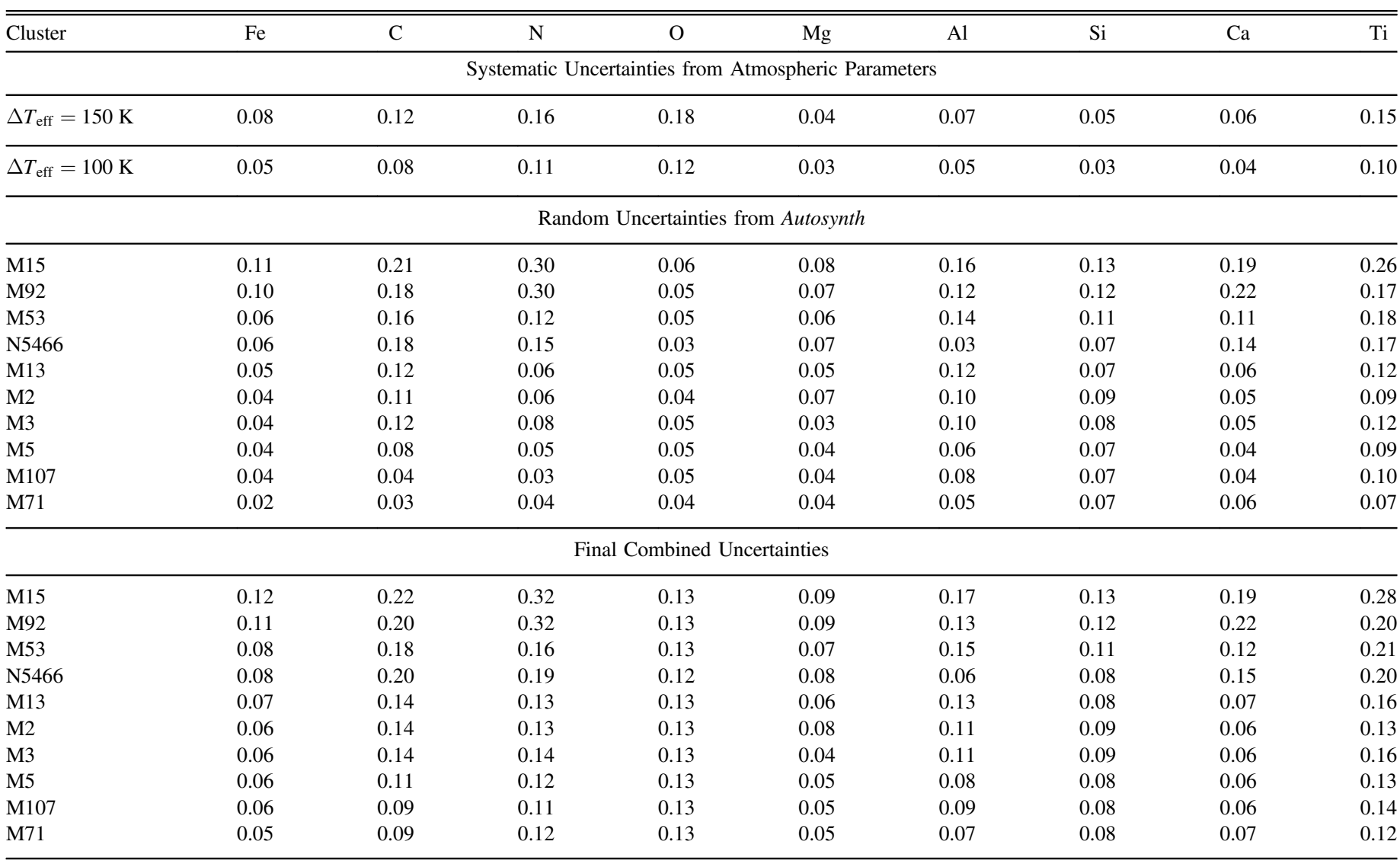

Note. Top panel: systematic uncertainty estimates from changes in $T_{\text {eff }}, \log g$, and $v_{\text {micro }}$. Middle panel: average random uncertainties reported by autosynth. Bottom panel: the final uncertainties are the sum of uncertainties in quadrature from the middle panel and uncertainties for $\Delta T_{\text {eff }}=100 \mathrm{~K}, \Delta \log g=0.3$, and $\Delta v_{\text {micro }}=0.1 \mathrm{~km} \mathrm{~s}^{-1}$.

two spectra slightly different from the original spectrum. Then, the fit of each line is repeated while keeping all other parameters unchanged. The average difference between these two new fits and the original best-fit spectrum is the defined uncertainty associated with the fit itself. This uncertainty estimate is mainly sensitive to variations of noise in the spectrum in the defined windows for the $\chi^{2}$ fit. If its value is close to 0 , then the $(1+\sigma) \times$ spectrum and $(1-\sigma) \times$ spectrum give very similar or the same abundances. This is expected when working at high $\mathrm{S} / \mathrm{N}$ and with a well defined continuum.

While this uncertainty estimation method is reliable in most cases, it has its limitations for very noisy spectra, very weak lines, or when abundances are near upper limits. Uncertainties are usually overestimated for noisy spectra, while they are underestimated for weak lines and upper limits. Thus, we decided to use one uncertainty from autosynth per element per cluster by simply averaging together all uncertainties reported by the program. This resulted in one uncertainty estimate for every element in each cluster. Autosynth uncertainties are listed in the middle section of Table 4. The final estimated uncertainties were calculated by adding together in quadrature the uncertainties for $100 \mathrm{~K}$ difference in temperature (also 0.3 dex systematic difference in $\log g$, and $0.1 \mathrm{~km} \mathrm{~s}^{-1}$ in microturbulent velocity), and the average autosynth values that estimate random uncertainties. These final estimations for each element per cluster are given in the bottom panel of Table 4.

\section{LITERATURE COMPARISONS}

We take $[\mathrm{X} / \mathrm{Fe}]$ abundance values for $\mathrm{C}, \mathrm{N}, \mathrm{O}, \mathrm{Mg}, \mathrm{Al}, \mathrm{Si}$, $\mathrm{Ca}$, and $\mathrm{Ti}$ from high-resolution spectroscopic studies in the literature as a point of comparison for our abundance determinations. We use the same literature sources as Mészáros et al. (2013), and added more recently published papers listed in Table 5. The derivation of stellar parameters $T_{\text {eff }}$ and $\log g$ are described in detail in Section 2 and they were also compared to the literature. Our goal for these comparisons is not to cross-calibrate our new abundance determinations with the literature; rather, we are looking for cases where our abundances are systematically different from the literature or particular clusters or elements for which our homogeneously observed and analyzed data set can clarify conflicts in the literature.

Cross-identification between the GC stars in the DR10 APOGEE data set and the literature was performed using the Simbad online service ${ }^{22}$, based on 2MASS identifiers and $(\alpha$, $\delta$ ) coordinates. Because there is a large and heterogeneous literature on chemical abundances in GC stars, we are providing our cross-identifications as a resource for the community. Table 5 lists 2MASS ID and position and alternate

${ }^{22}$ http://simbad.u-strasbg.fr/simbad/ 


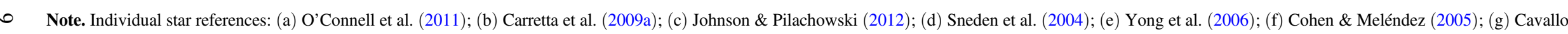

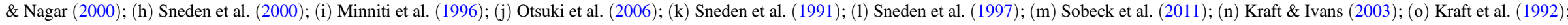

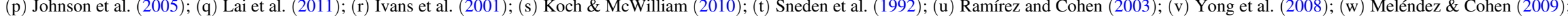

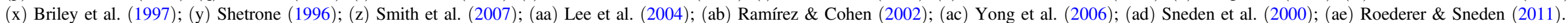

(This table is available in its entirety in machine-readable and Virtual Observatory (VO) forms.) 

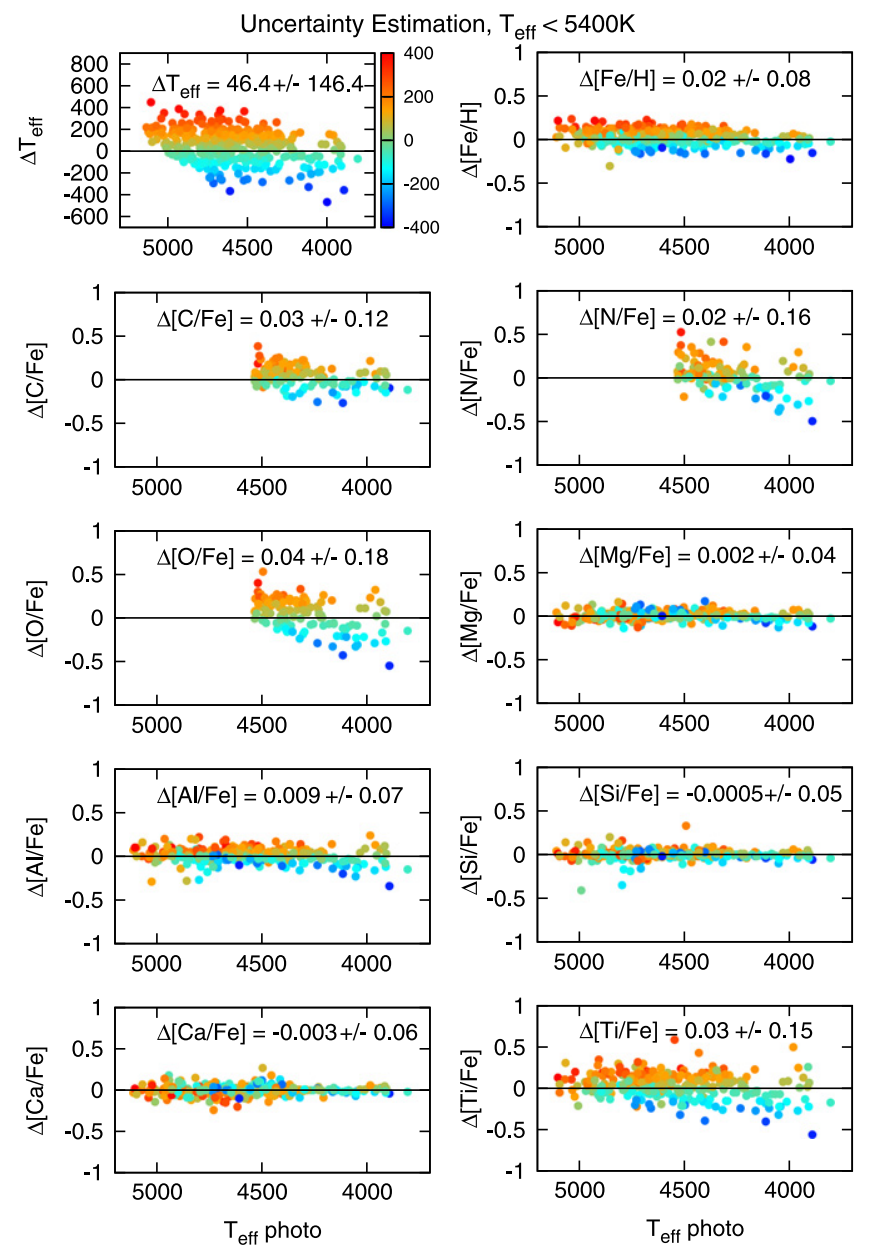

Figure 3. Differences in abundances produced by two runs adopting different temperatures: photometric and ASPCAP temperatures; otherwise the same calculation method was used. The points are color-coded by the differences between the photometric and ASPCAP temperatures. The \pm errors give the standard deviation around the mean of the differences.

stellar identifiers from literature abundance studies for all of the stars considered in this study.

Figure 4 shows comparisons of our stellar parameters and abundances against the literature values. Different GCs are represented by different colored points. In general, we find a systematic offset of $\sim 100 \mathrm{~K}$ between our photometric $T_{\text {eff }}$ values and the spectroscopic effective temperatures from the literature, with a reasonably small scatter. This is similar to the typical difference between spectroscopic and photometric temperatures reported by Mészáros et al. (2013). Because of the degeneracies in deriving stellar parameters, the slightly higher temperatures in the literature are accompanied by a systematic offset of $\sim+0.2$ dex in $\log g$.

There are a few systematic differences between our abundance results and those in the literature. These can mainly be traced back to a change in the solar abundance scale as derived by Asplund et al. (2005). As one example, the $[\mathrm{Fe} / \mathrm{H}]$ metallicities we derive are typically 0.1 dex higher than those from the literature, which is quite similar in magnitude to the change in $\mathrm{A}(\mathrm{Fe}) \odot$ from 7.52 (Anders \& Grevesse 1989) to 7.45 (Asplund et al. 2005). Also, the solar abundance of oxygen was revised significantly, from $\mathrm{A}(\mathrm{O})$. $\&$ Grevesse 1989) to 8.66 (Asplund et al. 2005). Since we use the more recent solar abundance values from Asplund et al. (2005) whereas our earlier literature sources do not, we expect the $\sim 0.3$ dex offset visible in Figure 4 . The systematic difference in $[\mathrm{N} / \mathrm{Fe}]$ abundance is also likely to be due to the updated solar abundances.

Carbon is the only element studied in this paper for which this explanation does not hold: although the solar abundance was revised down, from $\mathrm{A}(\mathrm{C}) \cdot 8.56$ (Anders \& Grevesse 1989) to 8.39 (Asplund et al. 2005), our $[\mathrm{C} / \mathrm{Fe}]$ values are typically lower than the literature values. Unfortunately, literature values are available for only a subset of our stars, which makes it difficult to verify precisely any systematic behavior.

In the other elements we consider in the present study, there is generally good agreement between our results and those from the literature, which is encouraging. However, we find significant differences in $\mathrm{Al}$ abundances in stars with belowsolar metallicities. This is mainly driven by stars in M3, where the $[\mathrm{Al} / \mathrm{Fe}]$ abundances from Johnson et al. (2005) are larger than in the other literature sources. We also see significant scatter in $[\mathrm{Ca} / \mathrm{Fe}]$ and $[\mathrm{Ti} / \mathrm{Fe}]$ at low abundances; this is likely caused by our abundance determination method having difficulty with weak lines.

The $[\mathrm{Mg} / \mathrm{Fe}]$ versus $[\mathrm{Al} / \mathrm{Fe}]$ relations in $\mathrm{M} 3, \mathrm{M} 13$, and M5 provide useful examples of how our new data set compares with the literature. In M3, our abundance determinations show a clear bimodality in the $\mathrm{Mg}-\mathrm{Al}$ distribution. The study of Cavallo \& Nagar (2000) found a similar bimodality, but Johnson et al. (2005) found a smooth distribution. In M13 we find an extended anticorrelation between $[\mathrm{Mg} / \mathrm{Fe}]$ and $[\mathrm{Al} / \mathrm{Fe}]$, and the literature abundances lie within it. We have seven stars in common with Sneden et al. (2004), and they span the full anticorrelation, while the three and two stars, respectively, which we have in common with Cohen \& Meléndez (2005) and Cavallo \& Nagar (2000) are consistent with our abundance results but happen to inhabit small regions of abundance space. M5 is a similar case, where our $[\mathrm{Al} / \mathrm{Fe}]$ values span a range of -0.3 to +1.1 dex, while the Carretta et al. (2009a) Al abundances are limited to between -0.2 and +0.7 dex. As in M13, our sample is larger than the literature sample, and covers the full RGB, while the literature sample does not fully span the parameter space.

\section{VARIATIONS IN INDIVIDUAL ELEMENT ABUNDANCES}

Although there are well-known abundance patterns within GCs, large homogeneous studies that include a wide range of abundances are rare. Since we can determine abundances of most of the light elements for the stars in our sample, we examine the behavior of the known abundance patterns across a wide range in cluster metallicity and search for unexpected variations in the $\alpha$-elements. In this section we focus on the range of abundance within each cluster, to separate real abundance variations, bimodalities, and trends from possible measurement errors. Table 6 lists the average and standard deviation for each elemental abundance in each cluster.

\subsection{Correlations with $T_{\text {eff }}$}

In Figures 5-7 we show all nine derived abundances as a function of effective temperature. From Figure 5 we conclude 
Table 6

Abundance Averages and Scatter

\begin{tabular}{|c|c|c|c|c|c|c|c|c|c|c|}
\hline Cluster & {$[\mathrm{Fe} / \mathrm{H}]$} & {$[\mathrm{C} / \mathrm{Fe}]$} & {$[\mathrm{N} / \mathrm{Fe}]$} & {$[\mathrm{O} / \mathrm{Fe}]$} & $\mathrm{A}(\mathrm{C}+\mathrm{N}+\mathrm{O})$ & {$[\mathrm{Mg} / \mathrm{Fe}]$} & {$[\mathrm{Al} / \mathrm{Fe}]$} & {$[\mathrm{Si} / \mathrm{Fe}]$} & {$[\mathrm{Ca} / \mathrm{Fe}]$} & {$[\mathrm{Ti} / \mathrm{Fe}]$} \\
\hline \multicolumn{11}{|c|}{ Averages } \\
\hline M15 & -2.28 & -0.41 & 0.95 & 0.54 & 7.09 & 0.11 & 0.34 & 0.44 & 0.16 & 0.15 \\
\hline M92 & -2.23 & -0.41 & 0.93 & 0.58 & 7.19 & 0.14 & 0.42 & 0.45 & 0.10 & 0.09 \\
\hline M53 & -1.95 & -0.50 & 1.06 & 0.56 & 7.49 & 0.11 & 0.37 & 0.41 & 0.23 & 0.28 \\
\hline N5466 & -1.82 & -0.56 & 0.84 & 0.63 & 7.60 & 0.14 & -0.24 & 0.29 & 0.04 & 0.29 \\
\hline M13 & -1.50 & -0.53 & 0.89 & 0.28 & 7.69 & 0.13 & 0.61 & 0.40 & 0.26 & 0.20 \\
\hline M2 & -1.49 & -0.48 & 0.90 & 0.41 & 7.76 & 0.26 & 0.45 & 0.35 & 0.24 & 0.27 \\
\hline M3 & -1.40 & -0.46 & 0.69 & 0.40 & 7.84 & 0.15 & 0.21 & 0.30 & 0.12 & 0.11 \\
\hline M5 & -1.24 & -0.46 & 0.76 & 0.27 & 7.85 & 0.23 & 0.36 & 0.34 & 0.20 & 0.26 \\
\hline M107 & -1.01 & -0.21 & 0.69 & 0.33 & 8.15 & 0.24 & 0.47 & 0.48 & 0.15 & 0.21 \\
\hline M71 & -0.68 & -0.10 & 0.91 & 0.51 & 8.65 & 0.38 & 0.51 & 0.39 & 0.21 & 0.42 \\
\hline \multicolumn{11}{|c|}{ Scatter } \\
\hline M15 & 0.10 & 0.13 & 0.35 & 0.19 & 0.14 & 0.24 & 0.52 & 0.16 & 0.25 & 0.08 \\
\hline M92 & 0.10 & 0.11 & 0.23 & 0.19 & 0.13 & 0.23 & 0.48 & 0.12 & 0.17 & 0.20 \\
\hline M53 & 0.07 & 0.16 & 0.21 & 0.06 & 0.09 & 0.08 & 0.51 & 0.05 & 0.17 & 0.13 \\
\hline N5466 & 0.08 & 0.01 & 0.10 & 0.04 & 0.08 & 0.06 & 0.35 & 0.09 & 0.25 & 0.14 \\
\hline M13 & 0.07 & $0.07^{\mathrm{a}}$ & 0.17 & 0.17 & 0.15 & 0.15 & 0.53 & 0.09 & 0.14 & 0.14 \\
\hline M2 & 0.08 & $0.05^{\mathrm{a}}$ & 0.15 & 0.21 & 0.23 & 0.07 & 0.51 & 0.06 & 0.12 & $0.08^{\mathrm{a}}$ \\
\hline M3 & 0.08 & $0.08^{\mathrm{a}}$ & 0.13 & 0.23 & 0.15 & 0.06 & 0.43 & 0.04 & 0.08 & 0.13 \\
\hline M5 & 0.08 & $0.13^{\mathrm{a}}$ & 0.17 & 0.27 & 0.19 & 0.09 & 0.34 & 0.07 & 0.11 & $0.12^{\mathrm{a}}$ \\
\hline M107 & 0.06 & 0.09 & 0.27 & 0.15 & 0.15 & $0.10^{\mathrm{a}}$ & 0.15 & 0.05 & 0.21 & 0.16 \\
\hline M71 & 0.07 & 0.13 & 0.32 & 0.09 & 0.13 & $0.05^{\mathrm{a}}$ & 0.08 & 0.05 & 0.10 & 0.09 \\
\hline
\end{tabular}

\footnotetext{
${ }^{a}$ Standard deviation around the linear fit.
}

Table 7

Averages of Populations

\begin{tabular}{|c|c|c|c|c|c|c|c|c|c|c|c|c|}
\hline Cluster & Pop. $^{a}$ & $N^{\mathrm{b}}$ & $R^{\mathrm{c}}$ & {$[\mathrm{Fe} / \mathrm{H}]$} & {$[\mathrm{C} / \mathrm{Fe}]$} & {$[\mathrm{N} / \mathrm{Fe}]$} & {$[\mathrm{O} / \mathrm{Fe}]$} & {$[\mathrm{Mg} / \mathrm{Fe}]$} & {$[\mathrm{Al} / \mathrm{Fe}]$} & {$[\mathrm{Si} / \mathrm{Fe}]$} & {$[\mathrm{Ca} / \mathrm{Fe}]$} & {$[\mathrm{Ti} / \mathrm{Fe}]$} \\
\hline \multirow[t]{2}{*}{ M15 } & 1 & 10 & 0.43 & -2.31 & -0.49 & 0.78 & 0.64 & 0.20 & -0.19 & 0.31 & 0.08 & 0.11 \\
\hline & 2 & 13 & 0.57 & -2.26 & -0.33 & 1.11 & 0.43 & 0.04 & 0.75 & 0.53 & 0.24 & 0.17 \\
\hline M92 & 1 & 14 & 0.30 & -2.23 & -0.42 & 0.89 & 0.67 & 0.33 & -0.23 & 0.41 & 0.08 & 0.09 \\
\hline \multirow[t]{2}{*}{ M53 } & 1 & 8 & 0.50 & -1.96 & -0.43 & 0.91 & 0.59 & 0.38 & -0.11 & 0.42 & 0.23 & 0.24 \\
\hline & 2 & 8 & 0.50 & -1.95 & -0.54 & 1.13 & 0.55 & 0.25 & 0.84 & 0.41 & 0.23 & 0.31 \\
\hline N5466 & 1 & 6 & $\ldots$ & -1.83 & -0.56 & 0.84 & 0.64 & 0.15 & -0.42 & 0.28 & 0.14 & 0.29 \\
\hline & 2 & 49 & 0.60 & -1.49 & -0.58 & 0.92 & 0.12 & 0.09 & 0.99 & 0.40 & 0.28 & 0.21 \\
\hline \multirow[t]{2}{*}{ M2 } & 1 & 7 & 0.39 & -1.46 & -0.45 & 0.75 & 0.56 & 0.30 & -0.10 & 0.35 & 0.19 & 0.26 \\
\hline & 2 & 11 & 0.61 & -1.50 & -0.49 & 1.01 & 0.30 & 0.24 & 0.79 & 0.35 & 0.27 & 0.27 \\
\hline \multirow[t]{2}{*}{ M3 } & 1 & 39 & 0.66 & -1.40 & -0.42 & 0.59 & 0.53 & 0.18 & -0.08 & 0.30 & 0.13 & 0.11 \\
\hline & 2 & 20 & 0.34 & -1.38 & -0.54 & 0.86 & 0.16 & 0.10 & 0.79 & 0.29 & 0.10 & 0.12 \\
\hline M5 & 1 & 60 & 0.49 & -1.24 & -0.41 & 0.62 & 0.41 & 0.24 & 0.06 & 0.34 & 0.20 & 0.24 \\
\hline & 2 & 4 & $\ldots$ & -0.69 & -0.17 & 1.16 & 0.49 & 0.44 & 0.53 & 0.38 & 0.21 & 0.45 \\
\hline
\end{tabular}

\footnotetext{
${ }^{\text {a }}$ Population 1 is denoted by red, while population 2 is denoted by blue in Figures 8-12.

${ }^{\mathrm{b}} N$ : the number of stars in each population.

${ }^{\mathrm{c}} R$ : the ratio of number of stars in a population and the overall number of stars analyzed.

${ }^{\mathrm{d}}$ Populations are found using $\mathrm{N}$ instead of $\mathrm{Al}$.
}

that we measure constant Fe abundances in all of the clusters. $\mathrm{Mg}$ and $\mathrm{Al}$ abundances show a large range of values in some clusters (as discussed in Section 2, and further in Section 6), but no significant trends with $T_{\text {eff }}$ except in M107 and M71.
This trend is very weak in M71, and data are consistent with showing no trend within the uncertainties. However, in M107 the trend is stronger. We currently do not fully understand where these small correlations come from. We suspect that this 

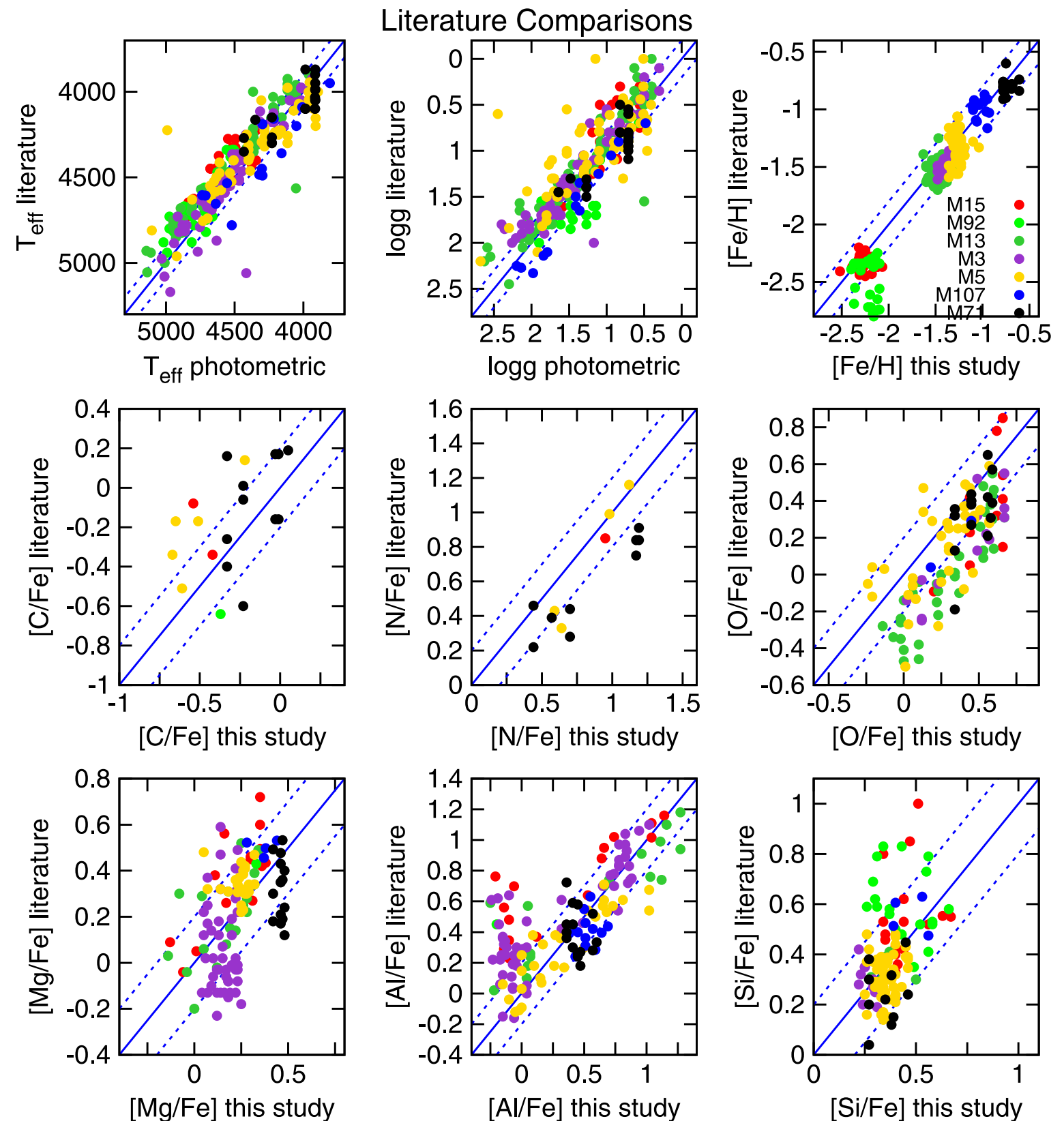

$[\mathrm{Mg} / \mathrm{Fe}]$ this study

$[\mathrm{Al} / \mathrm{Fe}]$ this study

[Si/Fe] this study
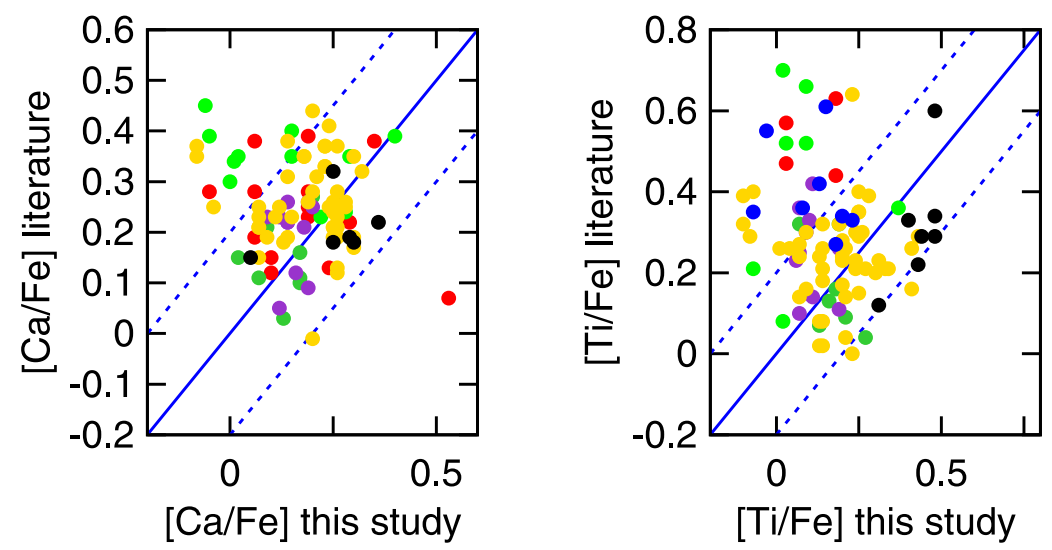

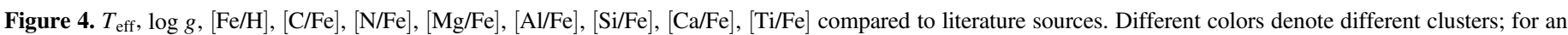

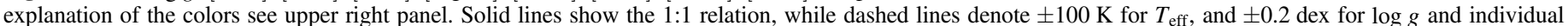
abundances. A detailed discussion can be found in Section 4. 
Table 8

Statistics of Correlations

\begin{tabular}{lrrrrrr}
\hline \hline Cluster & $\begin{array}{r}\Delta^{\mathrm{a}} \\
{[\mathrm{Mg} / \mathrm{Fe}]}\end{array}$ & $\begin{array}{r}\mathrm{e} \Delta^{\mathrm{b}} \\
{[\mathrm{Mg} / \mathrm{Fe}]}\end{array}$ & $\sigma^{\mathrm{c}}$ & $\begin{array}{r}\Delta \\
{[\mathrm{Si} / \mathrm{Fe}]}\end{array}$ & $\begin{array}{r}\mathrm{e} \Delta \\
{[\mathrm{Si} / \mathrm{Fe}]}\end{array}$ & $\sigma$ \\
\hline M15 & 0.16 & 0.037 & 4.3 & 0.22 & 0.055 & 4.0 \\
M92 & 0.28 & 0.029 & 9.7 & 0.06 & 0.038 & 1.6 \\
M53 & 0.13 & 0.035 & 3.7 & 0.01 & 0.055 & $<1$ \\
M13 & 0.12 & 0.014 & 8.6 & 0.02 & 0.018 & 1.1 \\
M2 & 0.06 & 0.038 & 1.6 & 0.00 & 0.043 & $<1$ \\
M3 & 0.08 & 0.011 & 7.3 & 0.01 & 0.024 & $<1$ \\
M5 & 0.03 & 0.008 & 3.8 & 0.01 & 0.014 & $<1$ \\
\hline
\end{tabular}

${ }^{\text {a }}$ Difference of the average $\mathrm{Mg}$ and $\mathrm{Si}$ abundance of populations.

$\mathrm{b}$ The estimated error of the difference.

${ }^{\mathrm{c}}$ Detection significance in $\sigma$.

is a result of a combination of effects. One such effect may be the use of model atmospheres that assume LTE, but that nonLTE effects (Bergemann \& Nordlander 2014) act to make the strong $\mathrm{Mg}$ lines in these metal-rich stars give the appearance of higher abundance in the cooler stars. Other possible effects are small systematic errors from estimating $T_{\text {eff }}$ and $\log g$ culminating during the synthesis. Abundances are also sensitive to the microturbulent velosity, so if the $\log g-v_{\text {micro }}$ expression used is not as accurate in this metallicity range, or the surface gravity is badly estimated, this propagates into abundances that are systematically off through $v_{\text {micro }}$.

The minimum $[\mathrm{C} / \mathrm{Fe}]$ (Figure 6) that can be measured from the $\mathrm{CO}$ lines strongly depends on temperature. Because RGB stars in GCs generally have low carbon abundances, we can only set upper limits for a number of our stars. Our $[\mathrm{C} / \mathrm{Fe}]$ values are an average of the derived abundances from five $\mathrm{CO}$ windows. As a result determining the upper limit is more challenging because $\mathrm{CO}$ lines in certain windows disappear faster with rising temperature than in others. We carefully checked every CO window fit and selected upper limits if the $\mathrm{CO}$ band head was not visible in more than three windows by looking at the flatness of the $\chi^{2}$ fit around the minimum value. Because the derived abundance of $\mathrm{N}$ from the $\mathrm{CN}$ lines is anticorrelated with the value of $[\mathrm{C} / \mathrm{Fe}]$ used, all stars with upper limits in $[\mathrm{C} / \mathrm{Fe}]$ have also lower limits in $[\mathrm{N} / \mathrm{Fe}]$. These upper limits for $[\mathrm{C} / \mathrm{Fe}]$ and lower limits for $[\mathrm{N} / \mathrm{Fe}]$ are identified with open triangles in all figures.

We see clear correlations between $[\mathrm{C} / \mathrm{Fe}]$ and $T_{\text {eff }}$ in $\mathrm{M} 13$, M3, M2, and M5 when omitting upper limits. We interpret these as a sign of "deep mixing," a nonconvective mixing process that causes steady depletion of surface carbon abundance and an enhancement in nitrogen abundance in all low-mass RGB stars (Gratton et al. 2004). We derive only upper limits on $[\mathrm{C} / \mathrm{Fe}]$ for M15, M92, M53, and NGC 5466 because the $\mathrm{CO}$ bands are quite weak at such low metallicity. With only upper limits, it is not possible to identify abundance trends in these clusters. However, previous studies (Martell et al. 2008; Shetrone et al. 2010; Angelou et al. 2012) have found signs of the same deep mixing process in action in those clusters.

The only cluster in which we see a sign of nitrogen enrichment with declining temperature is M13. Possible trends in nitrogen are not necessarily a result of astrophysics, because in our methodology small systematic errors in temperature can result in systematic errors in $\mathrm{N}$ abundance because deriving [N/ $\mathrm{Fe}]$ is challenging from the $\mathrm{CN}$ lines. Other than the relatively large errors of $\mathrm{N}$, one also has to be careful with possible correlations of $\mathrm{C}$ and $\mathrm{N}$ with temperature because they can be generated on the RGB as part of deep mixing and also in a previous stellar evolution event that are responsible for the pollution.

In Figure 7 there is a weak trend, on par with the error, visible in the $\mathrm{Si}$ abundance in M13, but not in the other clusters. While $\mathrm{Ca}$ does not show any correlation with temperature, it does show temperature-dependent scatter in most of the GCs. The three $\mathrm{Ca}$ lines used in our analysis are generally weak and get significantly weaker above $4700 \mathrm{~K}$, which leads to higher errors related to increased sensitivity to the uncertainty in the continuum placement. Titanium, unexpectedly, shows a decline with decreasing temperature in M2, M5 and (marginally) in M107 in Figure 7. We suspect that inaccuracies in our analysis of the hotter star spectra are driving this apparent trend since the $\mathrm{S} / \mathrm{N}$ is lower for those stars than for the cooler, brighter giants. Because these trends in $\mathrm{Mg}$ (Figure 5), Si, and Ti (Figure 7) only show up in a handful of clusters, we believe that they are a result of difficulties in data analysis for certain lines in certain stars and not any systematic mishandling in, e.g., $v_{\text {micro }}$ estimation.

We choose to fit these various observed trends with a linear equation, regardless of their origin, in order to explore the scatter around the trend. We fit lines to $[\mathrm{Mg} / \mathrm{Fe}]$ in M107 and $\mathrm{M} 71 ;[\mathrm{C} / \mathrm{Fe}]$ in $\mathrm{M} 13, \mathrm{M} 2, \mathrm{M} 3$, and M5 and $[\mathrm{Ti} / \mathrm{Fe}]$ in $\mathrm{M} 2$ and M5. We are only using these fits to determine the internal scatter, which we define as the standard deviation about the fitted line. Throughout the rest of this paper we use the abundance values directly, except when discussing the $\mathrm{CN}$ anticorrelations in Section 6.4.

\subsection{Scatter and Errors}

An internal abundance scatter significantly larger than the estimated errors suggests an astrophysical origin. In fact, significant scatter in $\mathrm{N}, \mathrm{O}, \mathrm{Mg}$, and $\mathrm{Al}$ is well documented in the literature (Gratton et al. 2012). The large star-to-star variations in these elements are the result of the $\mathrm{CNO}, \mathrm{Ne}-\mathrm{Na}$, and $\mathrm{Mg}-\mathrm{Al}$ cycles, which play an important role in the nuclear fusion processes at the early stages of RGB (CNO) and AGB $(\mathrm{Ne}-\mathrm{Na}, \mathrm{Mg}-\mathrm{Al})$ evolution. Figure 8 shows the internal scatter calculated as the standard deviation around the mean values (in some cases around a fitted linear equation, as explained earlier), and the final combined estimated uncertainties from Table 4, as a function of cluster average metallicity. We note that the small number of stars analyzed in NGC 5466 limits our ability to make a detailed analysis of $\mathrm{C}, \mathrm{N}$, and $\mathrm{O}$ in this cluster: because we were only able to measure the abundance of these elements in the three stars that have temperatures below $4500 \mathrm{~K}$, the scatter is unrealistically higher than the calculated uncertainty.

The star-to-star scatter in $[\mathrm{Fe} / \mathrm{H}]$ is quite similar to our measurement uncertainties, indicating that no significant metallicity variations in the clusters are detected in this study. Recently, Yong et al. (2014) have discovered three distinctive groups with different iron abundances in M2. The first group has a dominant peak at $[\mathrm{Fe} / \mathrm{H}]=-1.7$, while the second and third have smaller peaks at 1.5 and 1.0, but the membership for the latter group is not conclusive. We see no such behavior in M2. The discrepancy between these two studies is most likely the result of a selection effect. Yong et al. (2014) selected stars that belong to a second RGB (Lardo et al. 2012) within the 
$[\mathrm{Fe} / \mathrm{H}],[\mathrm{Mg} / \mathrm{Fe}],[\mathrm{Al} / \mathrm{Fe}]$ as a function of $\mathrm{T}_{\text {eff }}, \mathrm{T}_{\text {eff }}<5400 \mathrm{~K}$

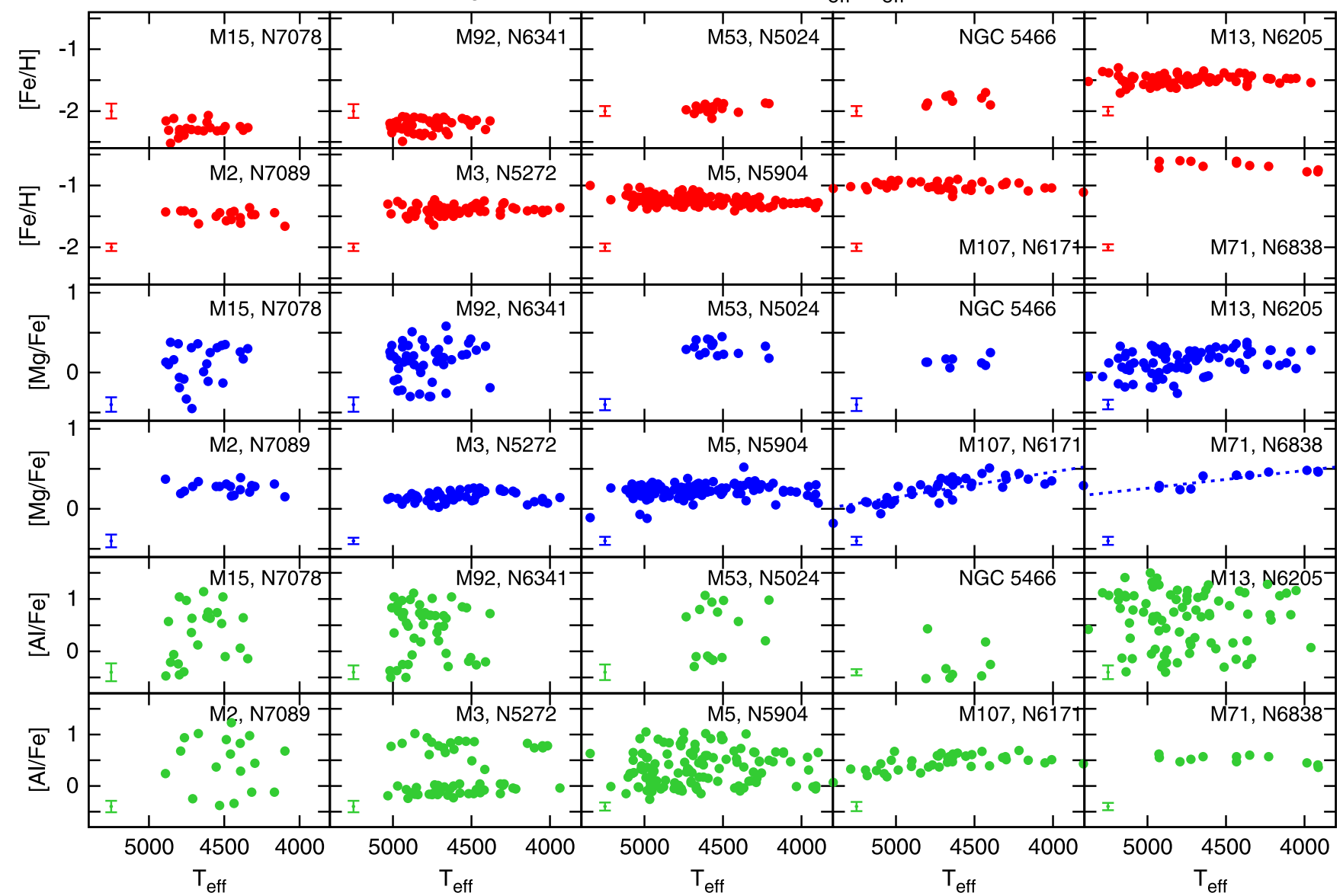

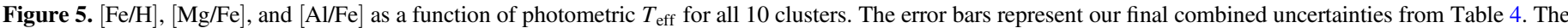
linear fit is plotted over $[\mathrm{Mg} / \mathrm{Fe}]$ for M107 and M71 to remove the visible linear trend (see Section 5.1 for discussion).

cluster, while our sample selection was based upon previous observations (Zasowski et al. 2013) and all of our stars belong to the main RGB of this cluster.

The $\alpha$-elements $\mathrm{Si}$ and $\mathrm{Ti}$ also show constant distribution, but the scatter in $[\mathrm{Ca} / \mathrm{Fe}]$ is larger than our measurement uncertainties for most clusters in our sample. We attribute this to an increasing inaccuracy in $[\mathrm{Ca} / \mathrm{Fe}]$ with rising temperature, as discussed in Section 5.1, and not a real range in calcium abundance. Little to no range in iron and $\alpha$-elements is what we expect for normal GCs, although the clusters in which these abundances do vary are an intriguing puzzle (see, e.g., Marino et al. 2013). M107 stands out as having the largest discrepancy between the scatter of $[\mathrm{Ca} / \mathrm{Fe}]$ and its estimated uncertainty. However, the large internal scatter is not related to astrophysics, but to spectral contamination from telluric $\mathrm{OH}$ lines. Due to the cluster's radial velocity, two of the three Ca lines lie on top of atmospheric $\mathrm{OH}$ lines. Because the telluric correction is not perfect, the $\mathrm{Ca}$ lines are compromised in a way that is not included in our uncertainty estimation.

We do see scatter above the level of the uncertainties in some of the light-element abundances. In the case of carbon, we find that the scatter around the overall carbon depletion trend for M13, M2, M3, and M5 (as shown by dashed lines in Figure 6) is fairly small, although our measurement uncertainties get quite large at low metallicity. We do not see clear carbon depletion in M107 or M71; however, as discussed in
Martell et al. (2008), the rate of carbon depletion due to deep mixing is lower in higher-metallicity stars.

The range in $[\mathrm{N} / \mathrm{Fe}]$ abundance (Figure 8, second panel from the top, in the left column) in M71, M107, M5, M3, M13, and M2 is clearly larger than the estimated uncertainties. However, the difference between the scatter and the uncertainty decreases with decreasing cluster metallicity, and below $[\mathrm{Fe} / \mathrm{H}]=-1.7$ (NGC 5466, M53, M92, and M15) our derived [N/Fe] values are all lower limits. The decreasing discrepancy is a result of larger uncertainties in more metal-poor clusters and the fact that the minimum $\mathrm{N}$ abundance of a cluster is slightly increasing as the average metallicity decreases (Figure 6), while the maximum $\mathrm{N}$ is constant above $[\mathrm{M} / \mathrm{H}]=-1.7$. The uncertainties in $\mathrm{M} 15$ and M92 are significantly larger than in other clusters, and our measurements are not precise enough to judge the amount of $\mathrm{N}$ enrichment in these clusters. Fitting the $\mathrm{CN}$ lines in our defined $\mathrm{CN}$ window is also challenging. This is because they are usually blended with other molecular lines and because $\mathrm{N}$ abundance strongly depends on the derived $\mathrm{C}$ abundance, which is an upper limit in many cases. A more detailed analysis of the $\mathrm{C}-\mathrm{N}$ anticorrelation and $\mathrm{N}$ bimodality can be found in Section 6.4.

Our uncertainties of the $[\mathrm{O} / \mathrm{Fe}]$ abundance (Figure 8) are fairly constant as a function of cluster metallicity. This is because the error is dominated by a strong sensitivity to the temperature used in the model atmosphere, and the internal scatter varies between clusters. Unfortunately, the $\mathrm{Na}$ lines 
[C/Fe], [O/Fe], [N/Fe] as a function of $\mathrm{T}_{\text {eff }}, \mathrm{T}_{\text {eff }}<4520 \mathrm{~K}$

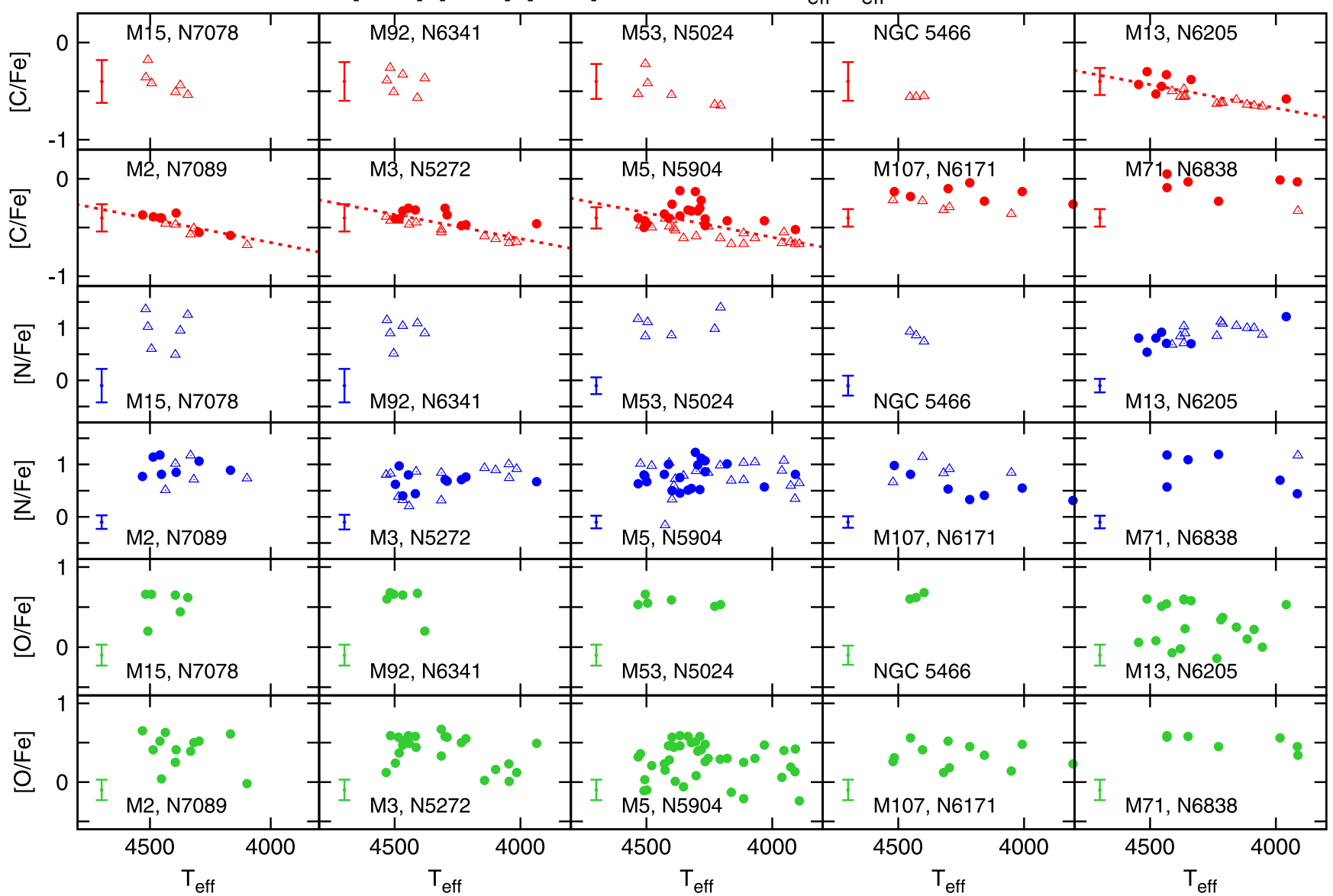

Figure 6. $[\mathrm{C} / \mathrm{Fe}],[\mathrm{N} / \mathrm{Fe}]$, and $[\mathrm{O} / \mathrm{Fe}]$ as a function of photometric $T_{\text {eff }}$ for all 10 clusters. Open triangles mark upper limits for $[\mathrm{C} / \mathrm{Fe}]$ and lower limits for $[\mathrm{N} / \mathrm{Fe}]$, while the real detections are plotted using filled red dots. The error bars represent our final combined uncertainties from Table 4 . The linear correlation in $[\mathrm{C} / \mathrm{Fe}]$ as a function of $T_{\text {eff }}$ in M13, M2, M3, and M5 is the effect of CNO burning on the RGB. The fitted lines are used to remove the trend in order to estimate the scatter in these clusters (see Section 5.1 for discussion).

available in the APOGEE spectra are too weak to investigate the $\mathrm{O}-\mathrm{Na}$ anticorrelation in our sample, so we can only limit our discussion to the scatter of $[\mathrm{O} / \mathrm{Fe}]$. We use the latter as an indication of the strength of $\mathrm{O}-\mathrm{Na}$ anticorrelation in a cluster. In M71 and M107 the $\mathrm{O}$ scatter is comparable to the uncertainties, suggesting that the $\mathrm{O}-\mathrm{Na}$ anticorrelation is not as extended as in the rest of the clusters. A clear spread is visible in M5, M3, M2, M13, M15, and M92, while in M53 the $\mathrm{O}$ abundances are constant and the scatter is significantly smaller than what is expected from the uncertainties.

The effects of the $\mathrm{Mg}-\mathrm{Al}$ cycle are very noticeable for all clusters except M71 and M107 (Figure 8, second panels from the bottom). The scatter in $\mathrm{Mg}$ abundance in those clusters is close to the estimated uncertainties after taking the linear correlations with $T_{\text {eff }}$ into account (Figure 5). In other clusters the scatter of $[\mathrm{Mg} / \mathrm{Fe}]$ also closely follows the uncertainties, except for M15 and M92 where the most Mg poor stars can be found. We see no Al enrichment in M107 and M71; however, as the average metallicity decreases, the amount of internal scatter rapidly starts to deviate from the error, and we see a large spread in $\mathrm{Al}$ in all other GCs. A more detailed analysis of the $\mathrm{Mg}-\mathrm{Al}$ anticorrelations and $\mathrm{Al}$ populations can be found in Sections 6.2 and 6.3 .

Based on the examination of the spread of $\mathrm{N}$ and $\mathrm{Al}$ abundances, we conclude that all clusters possess multiple stellar populations either based on $\mathrm{N}$ or $\mathrm{Al}$, or both. We examine these populations in more detail in the next section. The derived $\alpha$ element abundances are also fairly constant in all GCs presented here, similar to what is reported in the literature (Gratton et al. 2004).

\section{MULTIPLE POPULATIONS}

Most light elements show star-to-star variations in all GCs. These large variations are generally interpreted as the result of chemical feedback from an earlier generation of stars (Gratton et al. 2001; Cohen et al. 2002), rather than inhomogeneities in the original stellar cloud from which these stars formed. Thus, the current scenario of GC evolution generally assumes that more than one population of stars was formed in each cluster. The first generation of GC stars formed from gas that had been enriched by supernovae in the very early universe, while the second formed by combining gas from the original star-forming cloud with ejecta from the first generation stars. Only a fraction of first generation stars contribute to the pollution, and the timescale of the formation of these second generation stars depend on the nature of polluters; it is a couple of hundred $\mathrm{Myr}$ in the case of intermediate-mass AGB stars, but it is only a few Myr for fast rotating massive stars and massive binaries.

These first generation pollutors are thought to have been fast rotating massive stars (Decressin et al. 2007, intermediate mass $\left(M_{\text {star }}>3 M_{\odot}\right)$ AGB stars (D'Ercole et al. 2008), or massive 
$[\mathrm{Si} / \mathrm{Fe}],[\mathrm{Ca} / \mathrm{Fe}],[\mathrm{Ti} / \mathrm{Fe}]$ as a function of $\mathrm{T}_{\text {eff }}, \mathrm{T}_{\text {eff }}<5400 \mathrm{~K}$

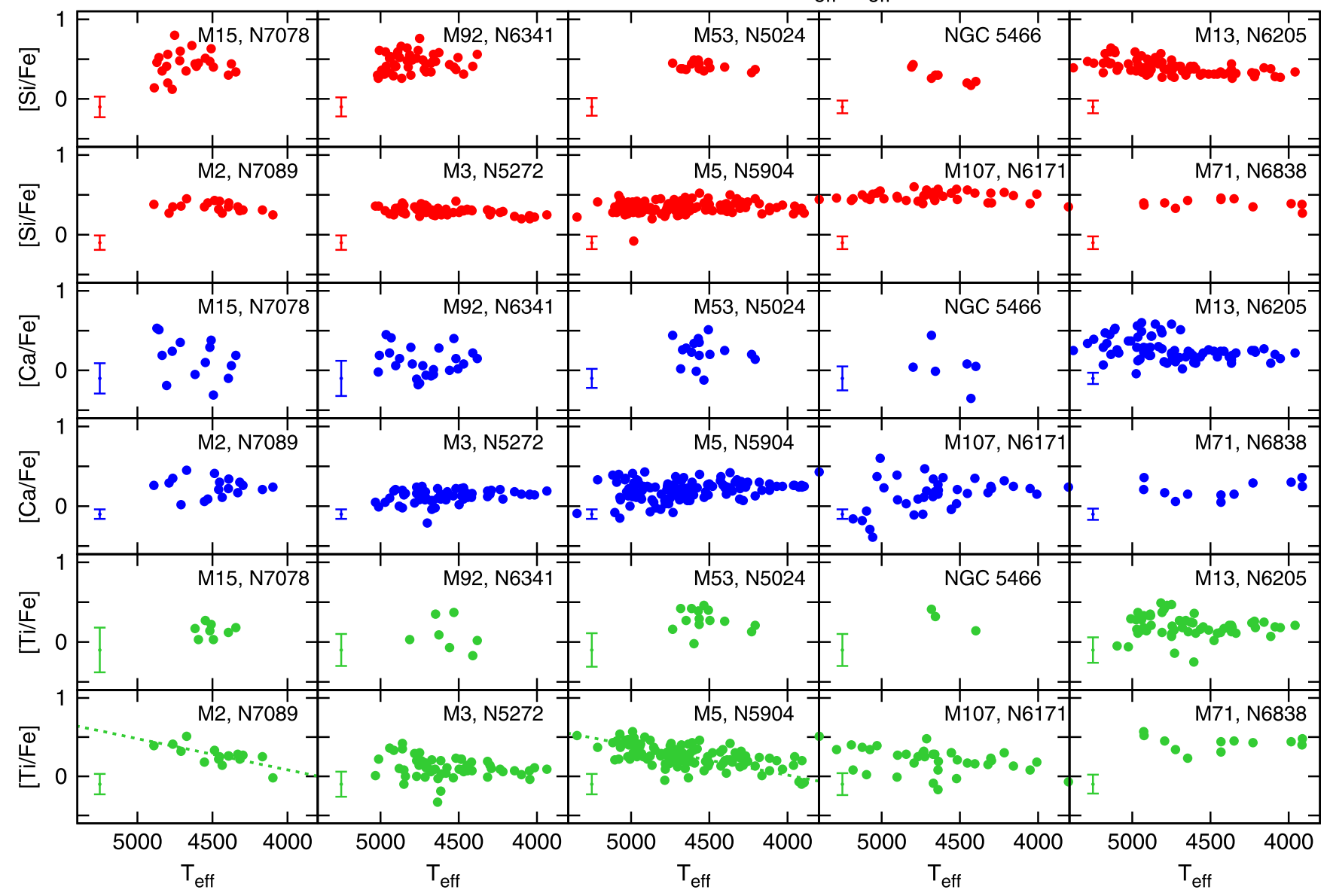

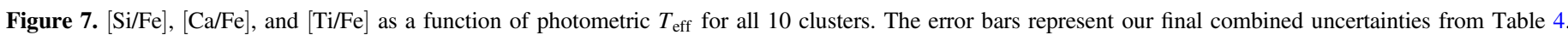
Trends in Ti for M2 and M5 are removed using the plotted lines when calculating the internal scatter (see Section 5.1 for discussion).

binaries (de Mink et al. 2009). Our data reveal the expected signatures of pollution from material enriched from the hot hydrogen burning cycles such as the $\mathrm{CNO}, \mathrm{NeNa}$, or $\mathrm{MgAl}$ cycles in all GCs in our sample. In this section we explore the various correlations between these elements, and we discuss individual star formation, and/or pollution events suggested by separate groups found in the $\mathrm{C}-\mathrm{N}$ and $\mathrm{Mg}-\mathrm{Al}$ anticorrelations.

\subsection{Identifying Multiple Populations}

To separate the various populations under study, we follow an approach similar to that of Gratton et al. (2011), who used $K$-means clustering (Steinhaus 1956) to identify multiple populations in $\omega \mathrm{Cen}$. Here, we use the extreme-deconvolution (XD) method of Bovy et al. (2011) $)^{23}$ to identify population groups and assign membership. This method fits the distribution of a vector quantity- here the elemental abundances- as a sum of $K$ Gaussian populations, whose amplitudes, centers, and covariance matrices are left entirely free. The algorithm can be applied to noisy or incomplete data, thus making the best possible use of all available data. In the current application, XD's main advantage is the latter since we are not able to measure abundances for all nine elements in all stars in our sample. Similar to the $K$-means, the number $K$ of populations to fit is an input to the

\footnotetext{
${ }^{23}$ Code available at http://github.com/jobovy/extreme-deconvolution.
}

algorithm; XD itself does not determine the optimal number of components to fit the distribution.

Briefly, the XD algorithm works by optimizing the likelihood of the Gaussian mixture model of the data. The optimization proceeds by an iterative procedure consisting of repeated expectation (E) and maximization (M) steps. In the $\mathrm{E}$ step, each datum is probabilistically assigned (a) membership in each population and (b) error-free values of each noisy or missing elemental abundance; in the M step, each Gaussian's parameters are updated using its members' mean abundances and covariance calculated from the error-free values obtained in the E step. These steps are repeated until the likelihood stops increasing to within a small tolerance. The algorithm is proven to increase the likelihood in each EM-step.

We analyze each GC in our sample using this algorithm, with the $[\mathrm{Mg} / \mathrm{Fe}],[\mathrm{Al} / \mathrm{Fe}],[\mathrm{Si} / \mathrm{Fe}],[\mathrm{Ca} / \mathrm{Fe}],[\mathrm{Ti} / \mathrm{Fe}]$ abundances as well as only $[\mathrm{Mg} / \mathrm{Fe}]$ and $[\mathrm{Al} / \mathrm{Fe}]$. We find that the two separate analyses provide nearly identical results, indicating that $\mathrm{Si}, \mathrm{Ca}$, and Ti do not drive the populations. For M71 and M107 we used $[\mathrm{N} / \mathrm{Fe}]$ instead of $[\mathrm{Al} / \mathrm{Fe}]$, because $\mathrm{Al}$ does not show any spread in these two clusters. We run XD using uncertainties given by the "final combined errors" section in Table 4, and also without any uncertainties, and found that the two different runs produce identical results. We include missing abundance measurements by using a large uncertainty for these measurements. Group membership for each star is 
Cluster internal scatter compared to uncertainties
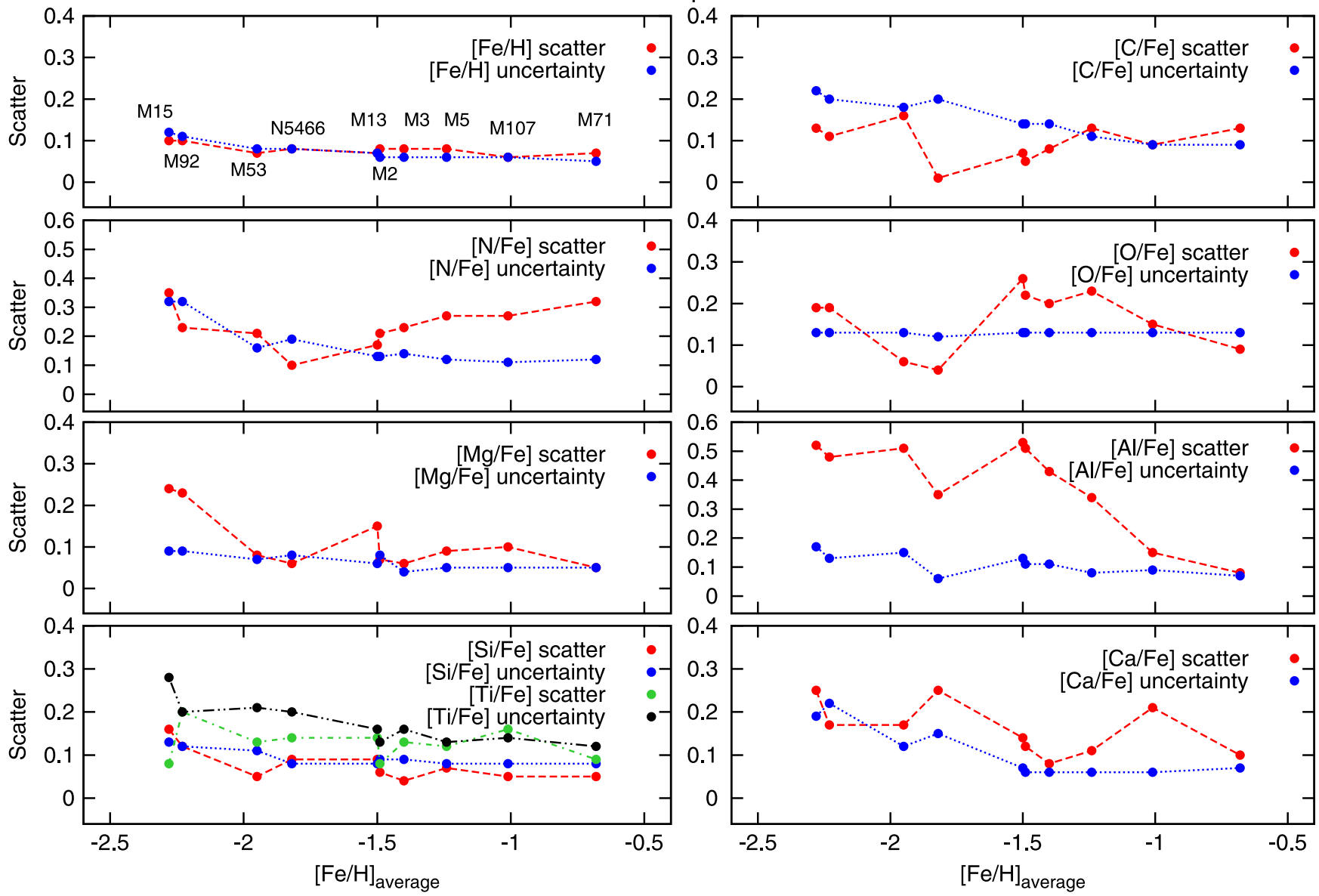

Figure 8. Internal scatter in each cluster from Table 6 (red dots) compared to our estimated final combined errors from Table 4 (blue dots).

determined using the best-fit Gaussian mixture by calculating the posterior probability for each star to be a member of each population based on its elemental abundances and their uncertainties; stars are then assigned to the population for which this probability is the largest. These membership assignments are typically unambiguous (probabilities $\gtrsim 99 \%$ in most cases), with only a few cases for which the maximum probability is below $90 \%$. The abundance averages of populations are listed in Table 7 . In the next few subsections we discuss the results from the population fitting in more detail for each individual cluster.

\subsection{The $M g-A l$ Anticorrelation}

In order for the $\mathrm{Mg}-\mathrm{Al}$ cycle to operate, high temperatures above 70 million K are required (Charbonnel \& Prantzos 2006). Because current cluster main-sequence stars are unable to reach these temperatures, the high $[\mathrm{Al} / \mathrm{Fe}]$ abundances we see in some GC stars imply that a previous generation of higher-mass or evolved stars must have contributed to their chemical composition.

Figure 9 shows $[\mathrm{Mg} / \mathrm{Fe}]$ versus $[\mathrm{Al} / \mathrm{Fe}]$ for all 10 clusters in our study. The two XD populations are plotted in red and blue, and a representative error bar is included in the top right corner of each panel. As noted previously, stars in M107 and M71 do not show a strong $\mathrm{Mg}-\mathrm{Al}$ anticorrelation, and their XD population assignments are based on $\mathrm{N}$ abundances. The light blue points in the panels for M107 and M71 are stars that are warmer than $4500 \mathrm{~K}$, and thus have no CNO measurements.

The extended distribution of $\mathrm{Mg}$ and $\mathrm{Al}$ abundances apparent in Figure 9 is typical for GCs, and it shows the influence of the $\mathrm{Mg}-\mathrm{Al}$ fusion cycle, which converts $\mathrm{Mg}$ into Al. However, there is a variety in the structure of this relationship that has not been thoroughly explored before: in some clusters (M92, M53, NGC 5466, M2, and M3), there are two distinct abundance groups with a gap, while in other clusters (M15, M13, and M5) there is no gap. This result strongly suggests that there is diversity in the process of stellar chemical feedback and star formation in GCs, which may relate to the larger environment in which they formed.

To date, the largest homogeneous study of $\mathrm{Mg}$ and $\mathrm{Al}$ abundances was carried out by Carretta et al. (2009a). We have several clusters in common, M15, M5, M107, and M71 which enables a direct comparison. M107 and M71 do not show any anticorrelation in either of the studies. Carretta et al. (2009a) only have upper limits for $\mathrm{Al}$ and $\mathrm{Mg}$ in M15, while we were able to make direct measurements, but even with upper limits the two studies show similar anticorrelations with largest spreads in both $\mathrm{Al}$ and $\mathrm{Mg}$ from the whole sample of clusters. The largest difference between the two studies can be seen in case of M5, where our values span a range of -0.3 to +1.1 dex, while the Al abundances of Carretta et al. (2009a) are limited to between -0.2 and 0.7 dex. The difference may be explained by a simple selection effect, as Carretta et al. (2009a) observed 


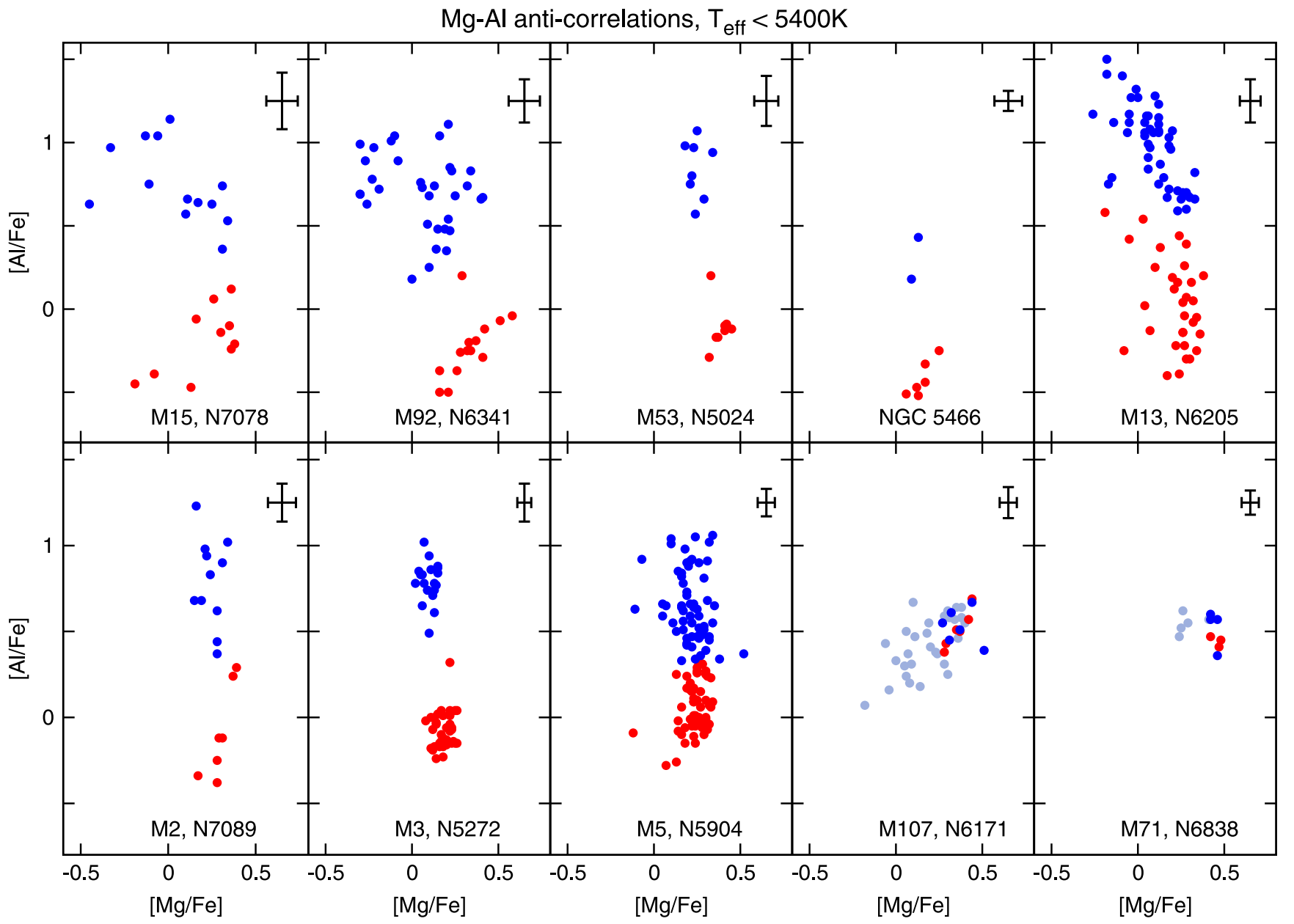

Figure 9. $\mathrm{Mg}-\mathrm{Al}$ anticorrelations. Colors mark the populations found with the $\mathrm{XD}$ code; the first population is denoted by red, and the second-generation denoted by blue points. Because $[\mathrm{N} / \mathrm{Fe}]$ was used to identify populations in M107 and M71, not all stars could be cataloged; these are denoted by light blue. The same colors denote the same stellar groupings in Figures 9-13.

13 stars in M5, while our sample size is 122 and covers almost the full extent of the RGB.

We discuss the extent of anticorrelation through differences in the average of the Mg abundance between the populations, divided by the estimated uncertainty in this average $\mathrm{Mg}$ difference. We use the final combined errors from Table 4. Table 8 lists the errors of the average differences with values of how much $\sigma$ detection is each difference. We find that in M15 $(4.3 \sigma), \quad \mathrm{M} 92(9.7 \sigma), \mathrm{M} 13(8.6 \sigma)$, and $\mathrm{M} 3(7.3 \sigma)$ the differences are statistically significant and the $\mathrm{Mg}-\mathrm{Al}$ anticorrelation exists. Two clusters, M53 (3.7 $\sigma)$ and M5 (3.8 $\sigma)$, also show large $\sigma$ detections, however, we would like to use a more conservative approach to the detection of anticorrelations out of a concern that our errors may be underestimated, given the degeneracies between stellar parameters and abundances in our analysis. M2 stands out as having no statistically significant $\mathrm{Mg}-\mathrm{Al}$ anticorrelation.

The summed abundance $\mathrm{A}(\mathrm{Mg}+\mathrm{Al})$ is expected to be constant as a function of $T_{\text {eff }}$ when material is completely processed through the $\mathrm{Mg}-\mathrm{Al}$ cycle, and that is what our results show in Figure 10. The only exception is M107, but the slight correlation is due to $\mathrm{Mg}$ correlating with temperature (see Figure 5), and this trend was not removed here.

$\mathrm{Al}$ is expected to correlate with elements enhanced by proton-capture reactions (N, Na; Figure 11) and anti- correlate with those depleted in H-burning at high temperature $(\mathrm{O}, \mathrm{Mg}$; Figure 11). The $\mathrm{Al}-\mathrm{O}$ anticorrelations and $\mathrm{Mg}-$ $\mathrm{O}$ correlations can be clearly seen in our data in Figure 11 for all clusters except M53, M107, and M71. The small number of stars observed in NGC 5466 does not allow us to investigate the correlations in that cluster in detail. However, the slight correlation of $\mathrm{Al}$ with $\mathrm{Si}$ that can be seen in M15 in Figure 11 is the evidence of ${ }^{28} \mathrm{Si}$ leaking from the $\mathrm{Mg}-\mathrm{Al}$ cycle, which is an intriguing result. Si participating in the light-element abundance pattern was first reported in NGC 6752 by Yong et al. (2005). Since then, Carretta et al. (2009a) have also found $\mathrm{Si}$ enhancement correlated with $\mathrm{Al}$ in NGC 2808. Those authors' interpretation was that it is only in low-metallilcity clusters, where the AGB stars burn slightly hotter, or in high-mass clusters, where the chemical enrichment is more efficient, that a $\mathrm{Si}$ enhancement will be observed in second-generation GC stars. The difference in $\mathrm{Si}$ abundance between the two XD-identified populations, listed in Table 8 , is significant relative to the error on that measurement. The Si-Al correlation in M15 is also accompanied by a $\mathrm{Si}-\mathrm{Mg}$ anticorrelation (Figure 11), which is further evidence of ${ }^{28} \mathrm{Si}$ being produced by hot bottom burning (HBB) in AGB stars (Karakas \& Lattanzio 2003). 


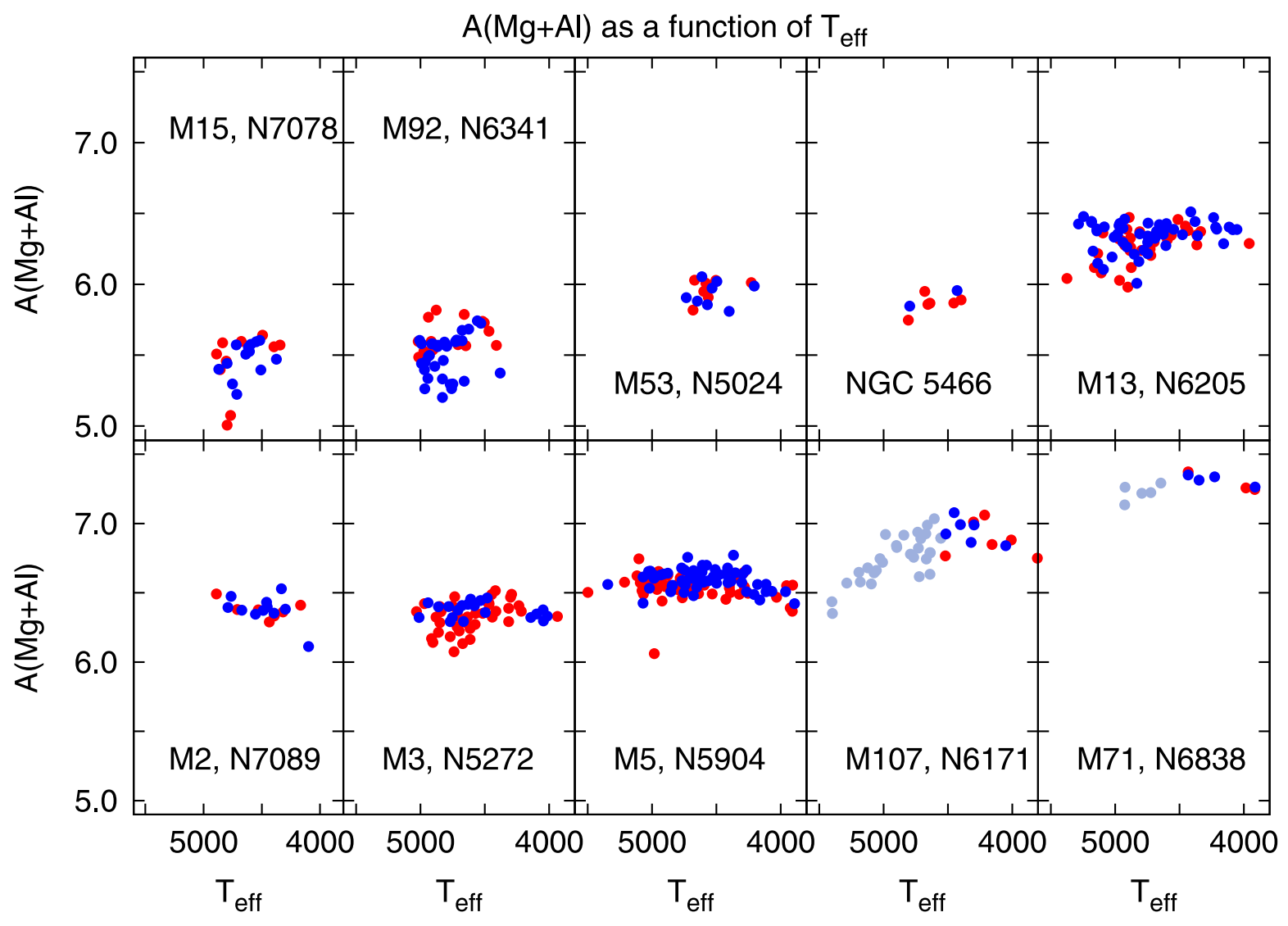

Figure 10. Combined abundance of $\mathrm{A}(\mathrm{Mg}+\mathrm{Al})$ as a function of effective temperature.

\subsection{The Spread of Al Abundances}

The spread of Al abundances (Figure 8) also increases significantly below $[\mathrm{Fe} / \mathrm{H}]=-1.1$, thus we can conclude that high mass, low metallicity AGB polluters that are able to process material at high, $60-70$ million $\mathrm{K}$, play an increasingly larger role in more metal-poor cluster. This behavior of $\mathrm{Al}$ abundances was previously observed by Carretta et al. (2009a), but the larger range of $\mathrm{Al}$ values presented in this paper makes this correlation clearer.

Theoretical AGB nucleosynthesis modeling indeed predicts this behavior (Ventura et al. 2001, 2013). The high-mass AGB stars reach higher temperatures at the bottom of the convective envelope; i.e., stronger $\mathrm{HBB}$ and advanced $(\mathrm{Mg}-\mathrm{Al})$ nucleosynthesis occurs with decreasing metallicity. We are seeing very advanced $\mathrm{Mg}-\mathrm{Al}$ nucleosynthesis in the most metal-poor clusters such as M15 and M92, while the most metal-rich clusters like M107 and M71 do not show this, as theoretically expected, if the high-mass AGB stars are the polluters. Also, this is corroborated by the Al-O anticorrelation; the most Alrich stars are O-poor showing the effects of very strong $\mathrm{HBB}$, because $\mathrm{HBB}$ proceeds completely and destroys $\mathrm{O}$.

An other related issue is that HBB is activated for lower masses with decreasing metallicity. High-mass and very lowmetallicity AGB stars do not exist in GCs today due to their extremely short lifetimes, but this trend seems to be confirmed both theoretically (Ventura et al. 2001, 2013) and observationally at least for solar metallicities down to those of the Magellanic Clouds. From the observational point of view, we know that high-mass AGB stars in the Small Magellanic Cloud $([\mathrm{Fe} /$ $\mathrm{H}]=-0.7)$ activate $\mathrm{HBB}$ for progenitor masses $\left(M>3 M_{\odot}\right)$ lower than their solar metallicity counterparts $\left(M>4 M_{\odot}\right)$ (García-Hernández et al. 2006, 2009). Thus, at the lowest metallicities in M15 and M92 we also would expect more HBB $\mathrm{AGB}$ stars (i.e., with several degrees of $\mathrm{HBB}$ and $\mathrm{Mg}-\mathrm{Al}$ nucleosynthesis), because the minimum stellar mass to activate the $\mathrm{HBB}$ process (and advanced $\mathrm{Mg}-\mathrm{Al}$ nucleosynthesis) decreases with decreasing metallicity. Thus, we conclude that more polluted material would be present at the lowest metallicities.

Because of these reasons, we believe that our results support and add some evidence to the high-mass AGBs as GCs polluters.

\section{4. $C-N$}

Carbon, nitrogen, and sometimes oxygen are influenced by two independent processes in GC giants: a primordial anticorrelation, with the same first generation sources as the $\mathrm{O}-\mathrm{Na}$ and $\mathrm{Mg}-\mathrm{Al}$ patterns and also stellar evolution, driven by circulation between the hydrogen-burning shell and the surface (Sweigart \& Mengel 1979; Angelou et al. 2012). As discussed in the previous section, we see large star-to-star variations in $\mathrm{N}$ abundance in all clusters; however, our inability to measure $\mathrm{C}$ abundances below $[\mathrm{Fe} / \mathrm{H}]=-1.7$ dex leads us to only having upper limits, and therefore we restrict the discussion of $\mathrm{C}-\mathrm{N}$ anticorrelations to the more metal-rich clusters.

Figure 12 shows $[\mathrm{N} / \mathrm{Fe}]$ versus $[\mathrm{C} / \mathrm{Fe}]$ for all clusters. Deep mixing has to be taken into account when discussing the $\mathrm{CN}$ anticorrelations, so in M13, M2, M3, and M5, where we see deep mixing clearly, the correlation of $[\mathrm{C} / \mathrm{Fe}]$ with $T_{\text {eff }}$ was removed. Because deep mixing is not visible in M107 and 
$\mathrm{Si}$ and $\mathrm{O}$ (anti-)correlations

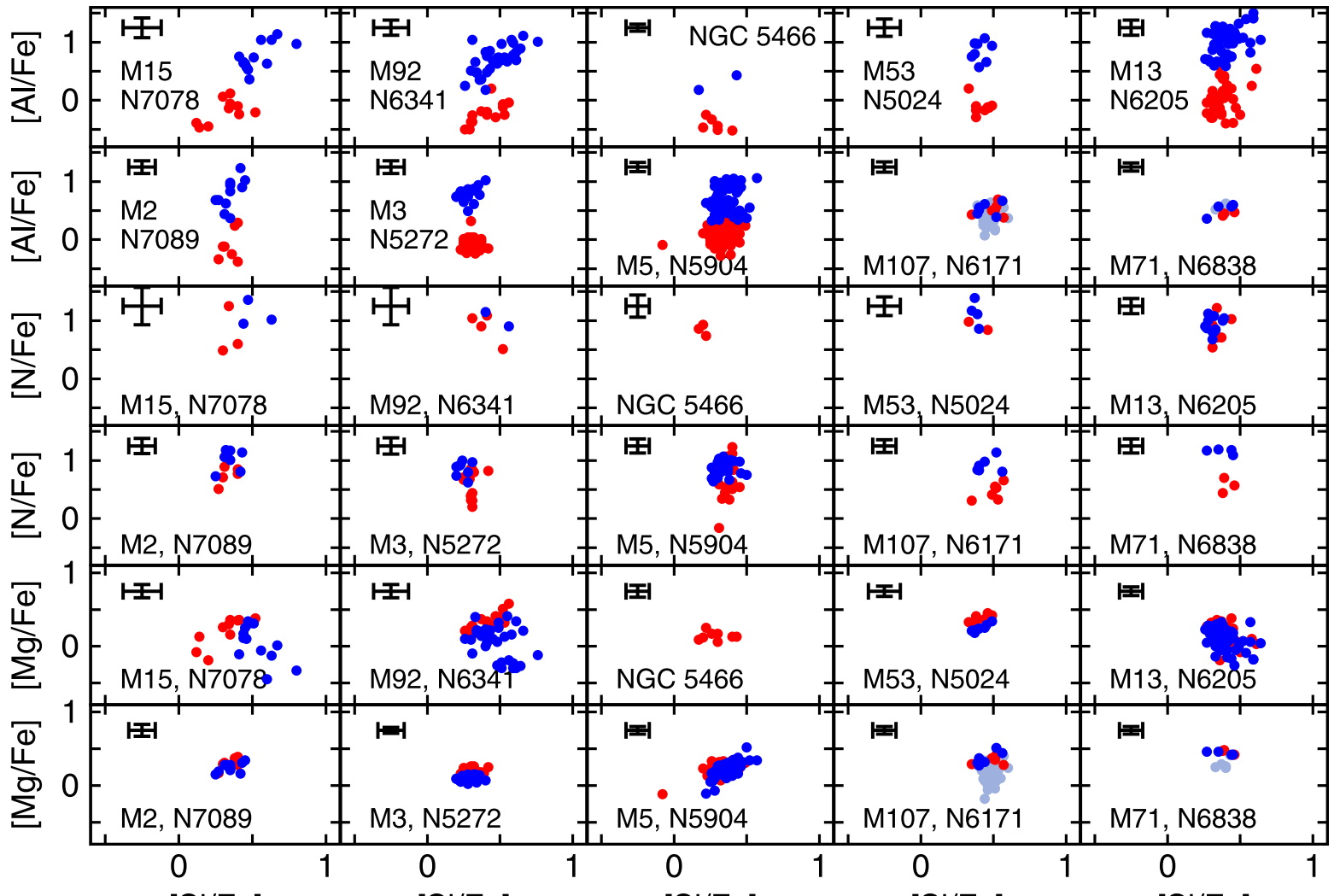

$[\mathrm{Si} / \mathrm{Fe}] \quad[\mathrm{Si} / \mathrm{Fe}] \quad[\mathrm{Si} / \mathrm{Fe}] \quad[\mathrm{Si} / \mathrm{Fe}] \quad[\mathrm{Si} / \mathrm{Fe}]$

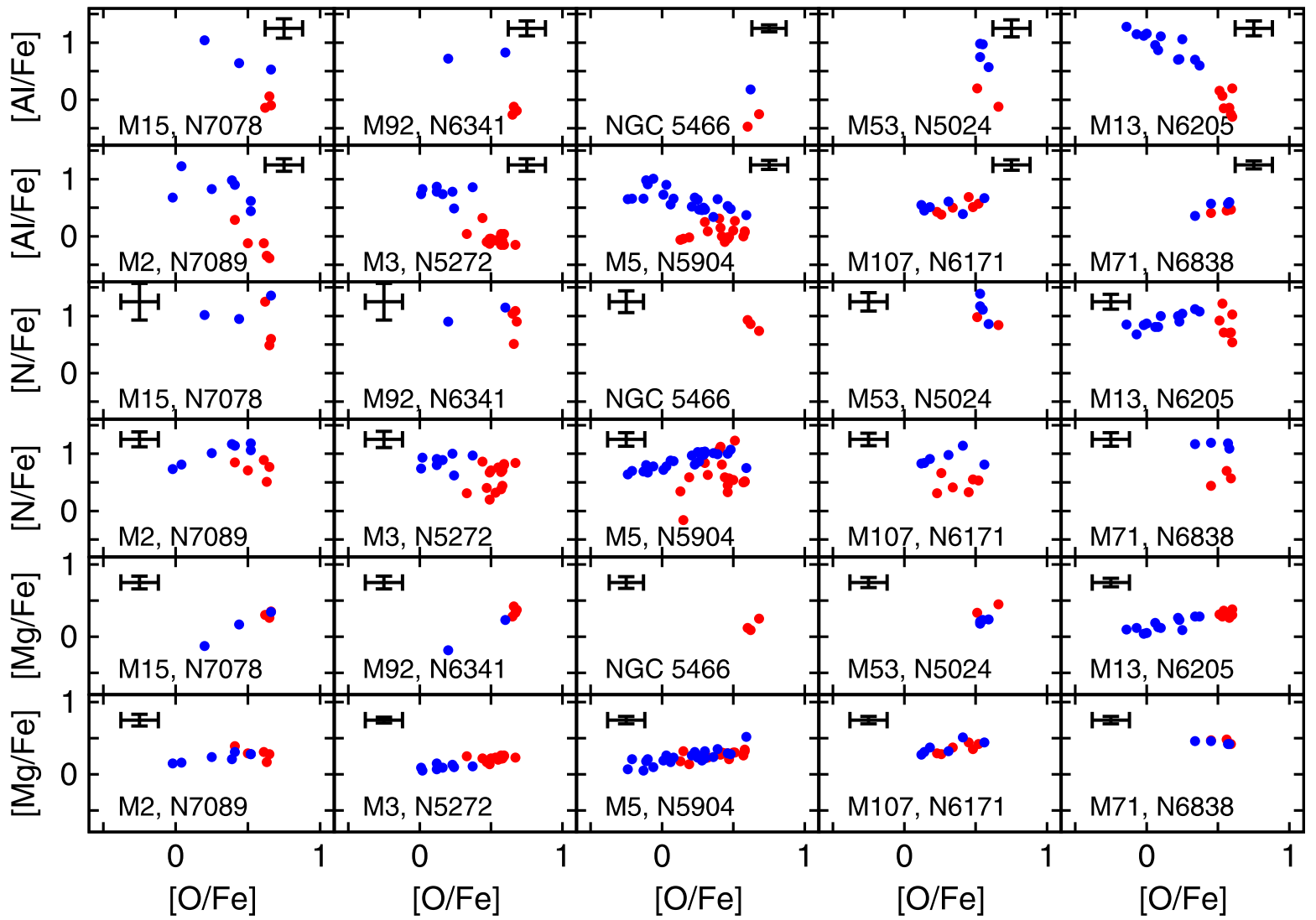

Figure 11. $\mathrm{Si}$ and $\mathrm{O}$ (anti)correlations with $\mathrm{Al}, \mathrm{N}$, and $\mathrm{Mg}$. For explanation of color coding, please see description of Figure 9. 


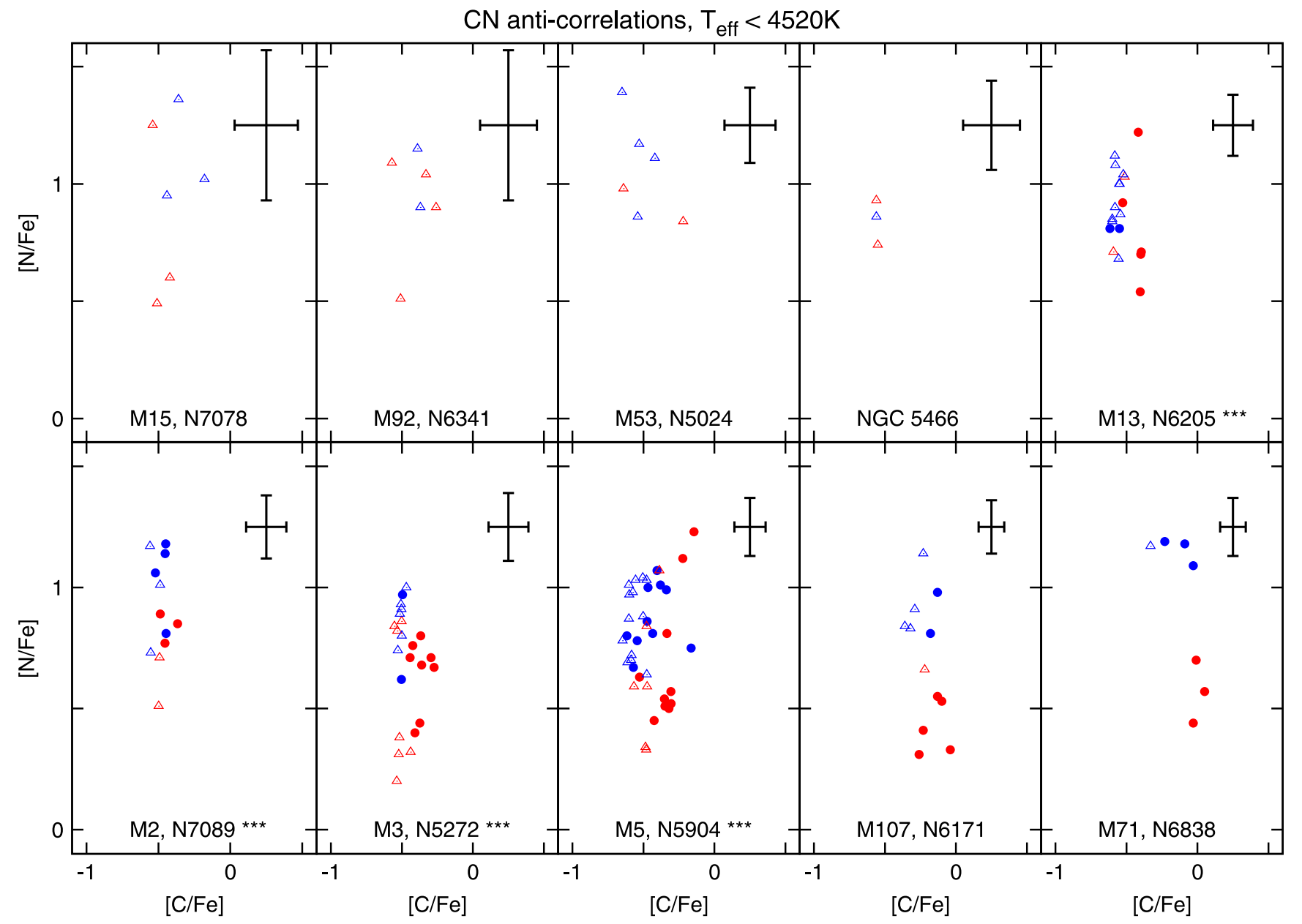

Figure 12. $\mathrm{CN}$ anticorrelations. Correlations of $[\mathrm{C} / \mathrm{Fe}]$ with temperature associated with deep mixing were removed in clusters marked by three stars. Upper limits are denoted by open triangles.

M71, we used the original values. A clear anticorrelation cannot be seen in any of the clusters. While M107 and M71 show weak anticorrelations, neither of these is statistically significant. The anticorrelation itself may be obscured by the relatively large errors for $[\mathrm{C} / \mathrm{Fe}]$ and $[\mathrm{N} / \mathrm{Fe}]$. As mentioned in Section 5.1, we see correlations of $[\mathrm{C} / \mathrm{Fe}]$ with temperature in M13, M3, M2, and M5, but these are not accompanied by increasing $[\mathrm{N} / \mathrm{Fe}]$ at the same time. We believe that this is due to our inability to measure $\mathrm{C}$ and $\mathrm{N}$ accurately: most of our abundance determinations are upper limits or close to them. If the anticorrelations exist, they must span ranges smaller than our uncertainties, which are always higher than 0.14 dex in $[C /$ $\mathrm{Fe}]$ and 0.12 dex in $[\mathrm{N} / \mathrm{Fe}]$. It is interesting to note that in $\mathrm{M} 3$, M107, and M71 there is a clear CN weak and CN strong group of stars, while in all more metal-poor clusters, $\mathrm{N}$ abundances fill out a broad distribution.

\section{5. $\mathrm{C}+\mathrm{N}+\mathrm{O}$}

We have $\mathrm{C}, \mathrm{N}$, and $\mathrm{O}$ abundances available for a large number of stars, thus we are able to investigate the $\mathrm{C}+\mathrm{N}+\mathrm{O}$ content in each cluster in detail. Studies from the literature showed that the $\mathrm{C}+\mathrm{N}+\mathrm{O}$ content in globular clusters are fairly constant to within 0.3 dex (Smith et al. 1996; Ivans et al. 1999; Carretta et al. 2005), except for N1851 where Yong et al. (2009) find a spread of 0.57 dex. According to Yong et al. (2009) the large spread in $\mathrm{C}+\mathrm{N}+\mathrm{O}$ in $\mathrm{N} 1851$ is probably attributed to larger than usual pollution from lower mass AGB stars than in other clusters, but these results were questioned by Villanova et al. (2010) as they did not see spread of $\mathrm{C}+\mathrm{N}+\mathrm{O}$ larger than 0.3 dex.

We also see near constant $\mathrm{C}+\mathrm{N}+\mathrm{O}$ in our sample, which is consistent with the material in these stars having undergone CNO cycling in the first-generation of stars in the RGB phase. In Figure 13 we show $\mathrm{A}(\mathrm{C}+\mathrm{N}+\mathrm{O})$ as a function of $T_{\text {eff }}$. The spread is significantly larger than what is reported in literature, between 0.4 and 0.6 dex for M13, M2, M3, and M5. The spread in these clusters is at the level of what has been found in N1851, but we think this is mostly associated with large uncertainties of $[\mathrm{N} / \mathrm{Fe}]$ measurements. We do not find clear separation in the amount of $\mathrm{A}(\mathrm{C}+\mathrm{N}+\mathrm{O})$ between the two populations found in any of the clusters.

As previously mentioned, there are currently two leading models to explain the nature of the polluters: the first assumes that intermediate-mass AGB stars that are thermally pulsing and undergoing $\mathrm{HBB}$ expel material to the intra-cluster medium by strong mass loss (Ventura et al. 2001), while the second assumes that the pollution comes from hot, fast rotating stars (Decressin et al. 2007). According to the first theory, intermediate-mass $\mathrm{HBB}$ AGB stars could produce or not produce large $\mathrm{C}+\mathrm{N}+\mathrm{O}$ variations (see, e.g., Yong et al. 2009 and references therein). This is because the $\mathrm{C}+\mathrm{N}+\mathrm{O}$ predictions (which basically depend on the number of third dredge-up 
$\mathrm{A}(\mathrm{C}+\mathrm{N}+\mathrm{O})$ as a function of $\mathrm{T}_{\text {eff }}$ and $[\mathrm{Al} / \mathrm{Fe}]$
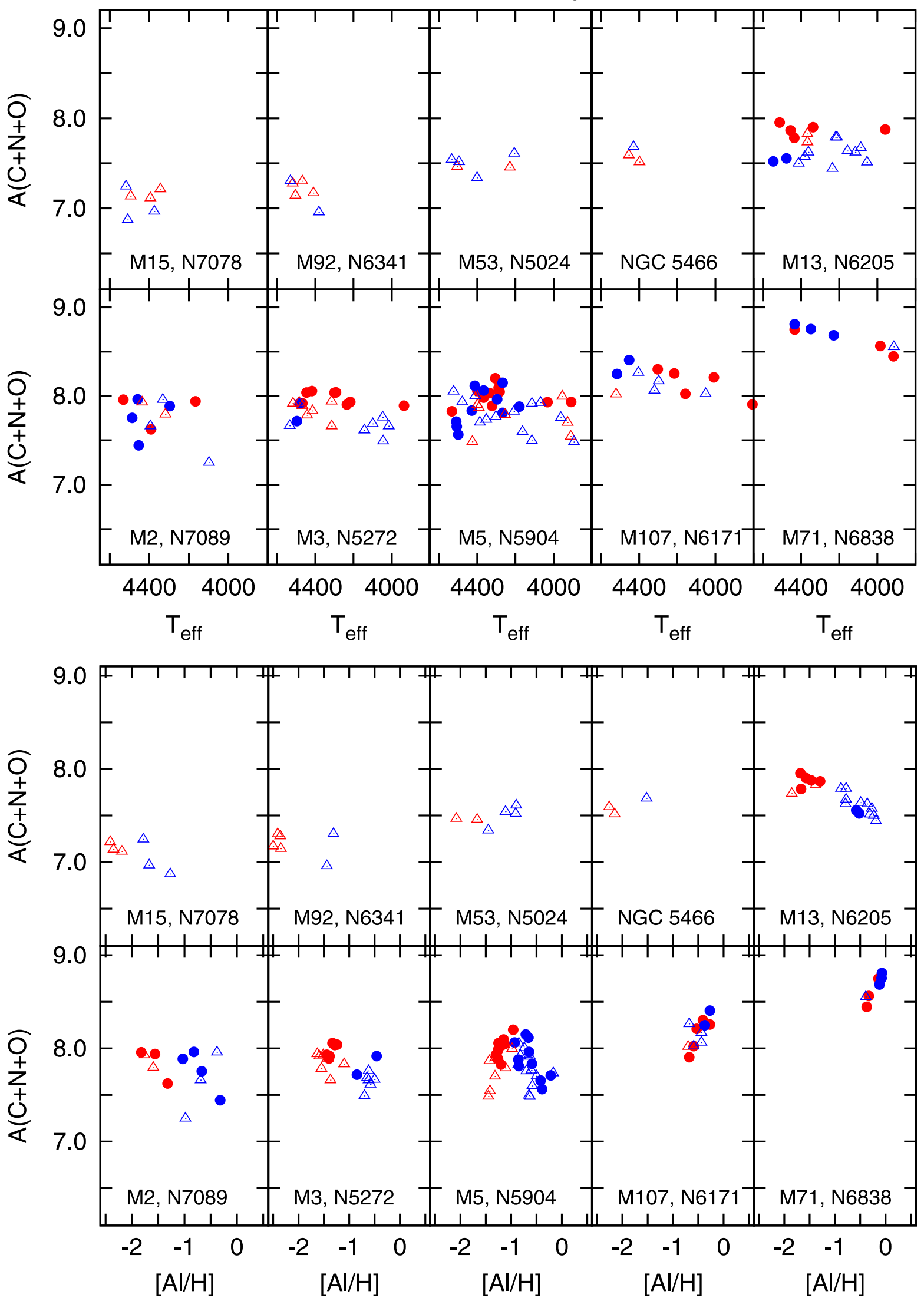

Figure 13. Upper panels: the sum of $\mathrm{C}, \mathrm{N}$, and $\mathrm{O}$ as a function of effective temperature. Upper limits are denoted by open triangles. For explanation of color coding, please see description of Figure 8. Lower panels: the sum of $\mathrm{C}, \mathrm{N}$, and $\mathrm{O}$ as a function of $[\mathrm{Al} / \mathrm{H}]$. A clear correlation is visible in $\mathrm{M} 13$, M2, M3, and M5, for more discussion see Section 6.4. 
episodes) for these stars are extremely dependent on the theoretical modeling, the convective model, and the mass loss prescription used (see, e.g., DAntona \& Ventura 2008; Karakas et al. 2012). A $(\mathrm{C}+\mathrm{N}+\mathrm{O})-\mathrm{Al}$ correlation is not clearly visible in the lower panel of Figure 10.

According to the first theory, AGB stars may explain a large spread in $\mathrm{A}(\mathrm{C}+\mathrm{N}+\mathrm{O})$ because they are expected to produce a substantial increase in the $\mathrm{C}+\mathrm{N}+\mathrm{O}$ abundance as they enhance $\mathrm{Na}$ and $\mathrm{Al}$ and deplete $\mathrm{O}$ and $\mathrm{Mg}$ (Yong et al. 2009). This should result in a $(\mathrm{C}+\mathrm{N}+\mathrm{O})-\mathrm{Al}$ correlation, which is not clearly visible in the lower panel of Figure 13. Weak correlations in M107 and M71, and weak anticorrelations in M13 and M3 are visible, but they are probably the result of large uncertainties in $\mathrm{C}$ and $\mathrm{N}$. More accurate measurements of $[\mathrm{N} / \mathrm{Fe}]$ will help to clarify the possible $(\mathrm{C}+\mathrm{N}+\mathrm{O})-\mathrm{Al}$ correlation.

\section{SUMMARY}

We investigated the abundances of nine elements for 428 stars in 10 GCs using APOGEE DR10 spectra. A homogeneous analysis of these GCs has not been accomplished previously, something that APOGEE is uniquely able to do because it can observe all the bright GCs in the northern hemisphere. A semi-automated code called autosynth was developed to provide abundances independent of those derived by ASPCAP. Our main goal was to examine the stellar populations in each cluster using a homogeneous data set. Based on our abundances, we find the following:

1. From the examination of the star-to-star scatter in $\alpha$ element abundance, only $\mathrm{O}$ and $\mathrm{Mg}$ show an abundance scatter larger than our measurement errors. However, this is expected, because $\mathrm{O}$ and $\mathrm{Mg}$ are part of the wellknown GC light-element abundance anticorrelations.

2. Population analysis using $[\mathrm{N} / \mathrm{Fe}]$ for M107 and M71 and $[\mathrm{Al} / \mathrm{Fe}]$ in the rest of the clusters confirms that each cluster can be divided into two discrete populations, though in M107 and M71 that division is clearest in the $\mathrm{N}-\mathrm{Al}$ plane, whereas in the other clusters it is best made in the $\mathrm{Mg}-\mathrm{Al}$ plane.

3. The anticorrelation of $\mathrm{Mg}$ and $\mathrm{Al}$ is clearly shown in M15, M92, M13, and M3, and to a lesser extent in M53 and M5. Interestingly, M2, which is also a metal-poor cluster, does not have a $\mathrm{Mg}-\mathrm{Al}$ anticorrelation. The increased number of stars in our sample compared to the literature enables us to discover more Al-rich stars, making the anticorrelation clearer in this data set than in previous studies.

4. The spread of $\mathrm{Al}$ abundances increases with decreasing cluster metallicity. This is in agreement with theoretical AGB nucleosynthesis predictions by Ventura et al. (2001, 2013). This suggests that high-mass HBB AGB stars, which are able to enrich $\mathrm{Al}$ while destroying $\mathrm{O}$, are more common polluters in metal-poor clusters than in metalrich ones.

5. A correlation between $\mathrm{Al}$ and $\mathrm{Si}$ in $\mathrm{M} 15$ indicates particularly high-temperature hydrogen burning in the stars that contributed the abundance pattern of the second generation stars. This is consistent with $\mathrm{Si}$ enhancement in GC stars as discussed in the literature, where it appears to be confined to low-metallicity and high-mass clusters.

6. Besides accessing northern GCs, APOGEE is also unique among other spectroscopic surveys because it is capable of measuring abundances of CNO. The total abundance of $\mathrm{C}+\mathrm{N}+\mathrm{O}$ is found to be constant in each cluster, within our errors. Our data set does not show an unambiguous correlation between $\mathrm{A}(\mathrm{C}+\mathrm{N}+\mathrm{O})$ and $[\mathrm{Al} / \mathrm{H}]$. In principle, this finding is not against the idea of intermediate-mass HBB AGB stars as the primary source of chemical selfenrichment in globular clusters. However, more precise measurements of $\mathrm{C}$ and $\mathrm{N}$ abundances will clarify this issue, and we expect that ASPCAP will be able to provide these in the future.

Szabolcs Mészáros is especially grateful for the technical expertise and assistance provided by the Department of Astronomy at Indiana University. We thank Eileen D. Friel, Catherine A. Pilachowski, and Enrico Vesperini for their detailed comments during the development of autosynth. Sarah Martell acknowledges the support of the Australian Research Council through DECRA Fellowship DE140100598. Sara Lucatello acknowledges partial support from grant PRIN MIUR 2010-2011 "Chemical and dynamical evolution of the Milky Way and Local Group Galaxies." Jo Bovy was supported by NASA through Hubble Fellowship grant HSTHF-51285.01 from the Space Telescope Science Institute, which is operated by the Association of Universities for Research in Astronomy, Incorporated, under NASA contract NAS5-26555. Timothy C. Beers acknowledges partial support for this work from grants PHY 08-22648; Physics Frontier Center/Joint Institute or Nuclear Astrophysics (JINA), and PHY 14-30152; Physics Frontier Center/JINA Center for the Evolution of the Elements (JINA-CEE), awarded by the US National Science Foundation. Katia Cunha acknowledges support for this research from the National Science Foundation (AST-0907873). Verne V. Smith acknowledges partial support for this research from the National Science Foundation (AST1109888). D. A. García-Hernández and Olga Zamora acknowledge support provided by the Spanish Ministry of Economy and Competitiveness under grant AYA-2011-27754. Funding for SDSS-III has been provided by the Alfred P. Sloan Foundation, the Participating Institutions, the National Science Foundation, and the U.S. Department of Energy Office of Science. The SDSS-III website is http://sdss3.org/. SDSS-III is managed by the Astrophysical Research Consortium for the Participating Institutions of the SDSS-III Collaboration including the University of Arizona, the Brazilian Participation Group, Brookhaven National Laboratory, University of Cambridge, Carnegie Mellon University, University of Florida, the French Participation Group, the German Participation Group, Harvard University, the Instituto de Astrofisica de Canarias, the Michigan State/Notre Dame/JINA Participation Group, Johns Hopkins University, Lawrence Berkeley National Laboratory, Max Planck Institute for Astrophysics, New Mexico State University, New York University, Ohio State University, Pennsylvania State University, University of Portsmouth, Princeton University, the Spanish Participation Group, University of Tokyo, University of Utah, Vanderbilt University, University of Virginia, University of Washington, and Yale University.

\section{REFERENCES}

Ahn, C. P., Alexandroff, R., Allende Prieto, C., et al. 2014, ApJS, 211, 17 Alam, S., Albareti, F. D., Allende Prieto, C., et al. 2015, arXiv:1501.00963 Anders, E., \& Grevesse, N. 1989, GeCoA, 53, 197 
Angelou, G. C., Stancliffe, R. J., Church, R. P., Lattanzio, J. C., \& Smith, G. H. 2012, ApJ, 749, 128

Asplund, M., Grevesse, N., \& Sauval, A. J. 2005, ASPC, 336, 25

Bastian, N., Lamers, H. J. G. L. M., de Mink, S. E., et al. 2013, MNRAS, 436, 2398

Bergemann, M., \& Nordlander, T. 2014, arXiv:1403.3088B

Bertelli, G., Girardi, L., Marigo, P., \& Nasi, E. 2008, A\&A, 484, 815

Bertelli, G., Nasi, E., Girardi, L., \& Marigo, P. 2009, A\&A, 508, 355

Borucki, W. J., Koch, D., Basri, G., et al. 2010, Sci, 327, 977

Bovy, J., Hogg, D. W., \& Roweis, S. T. 2011, AnApS, 5, 1657

Briley, M. M., Smith, V. V., King, J., \& Lambert, D. L. 1997, AJ, 113, 306

Briley, M. M., Smith, V. V., Suntzeff, N. B., et al. 1996, Natur, 383, 604

Cassisi, S., Salaris, M., Pietrinferni, A., et al. 2008, ApJL, 672, L115

Carretta, E., Bragaglia, A., Gratton, R., D’Orazi, V., \& Lucatello, S. 2009c, A\&A, 508, 695

Carretta, E., Bragaglia, A., Gratton, R., \& Lucatello, S. 2009a, A\&A, 505, 139

Carretta, E., Bragaglia, A., Gratton, R. G., et al. 2009b, A\&A, 505, 117

Carretta, E., Gratton, R. G., Lucatello, S., Bragaglia, A., \& Bonifacio, P. 2005 A\&A, 433, 597

Cavallo, R. M., \& Nagar, N. M. 2000, AJ, 120, 1364

Charbonnel, C., \& Prantzos, N. 2006, arXiv:astro-ph/0606220

Cohen, J. G., Briley, M. M., \& Stetson, P. B. 2002, AJ, 123, 2525

Cohen, J. G., \& Meléndez, J. 2005, AJ, 129, 303

Decressin, T., Meynet, G., Charbonnel, C., Prantzos, N., \& Ekström, S. 2007, A\&A, 464, 1029

DAntona, F., Bellazzini, M., Caloi, V., et al. 2005, ApJ, 631, 868

DAntona, F., \& Ventura, P. 2008, Msngr, 134, 18

D’Ercole, A., Vesperini, E., D’Antona, F., McMillan, S. L. W., \& Recchi, S. 2008, MNRAS, 391, 825

Eisenstein, D. J., Weinberg, D. H., Agol, E., et al. 2011, AJ, 142, 72

Epstein, C. R., Elsworth, Y. P., Johnson, J. A., et al. 2014, ApJL, 785, L28

Freeman, K. C. 2012, in ASP Conf. Ser. 458, Galactic Archaeology: NearField Cosmology and the Formation of the Milky Way, ed. W. Aoki et al. (San Francisco, CA: ASP), 393

García-Hernández, D. A., García-Lario, P., Plez, B., et al. 2006, Sci, 314, 1751

García-Hernández, D. A., Manchado, A., Lambert, D. L., et al. 2009, ApJ, 705, L31

Gilmore, G., Randich, S., Asplund, M., et al. 2012, Msngr, 147, 25

González Hernández, J. I., \& Bonifacio, P. 2009, A\&A, 497, 497

Gratton, R., Sneden, C., \& Carretta, E. 2004, ARA\&A, 42, 385

Gratton, R. G., Bonifacio, P., Bragaglia, A., et al. 2001, A\&A, 369, 87

Gratton, R. G., Carretta, E., \& Bragaglia, A. 2012, A\&ARv, 20, 50

Gratton, R. G., Johnson, C. I., Lucatello, S., et al. 2011, A\&A, 534, A72

Gunn, J. E., Siegmund, W. A., Mannery, E. J., et al. 2006, AJ, 131, 2332

Holtzman, J. A., Shetrone, M., Johnson, J. A., et al. 2015, arXiv:1501.04110

Ivans, I. I., Kraft, R. P., Sneden, C. S., et al. 2001, AJ, 122, 1438

Harris, W. E. 1996, AJ, 112, 1487

Ivans, I. I., Sneden, C., Kraft, R. P., et al. 1999, AJ, 118, 1273

Johnson, C. I., Kraft, R. P., Pilachowski, C. A., et al. 2005, PASP, 117, 1308

Johnson, C. I., \& Pilachowski, C. A. 2012, ApJL, 754, L38

Karakas, A. I., García-Hernández, D. A., \& Lugaro, M. 2012, ApJ, 8, 751

Karakas, A. I., \& Lattanzio, J. C. 2003, PASA, 20, 393

Koch, A., \& McWilliam, A. 2010, AJ, 139, 2289

Kraft, R. P. 1994, PASP, 106, 553

Kraft, R. P., \& Ivans, I. I. 2003, PASP, 115, 143

Kraft, R. P., Sneden, C., Langer, G. E., \& Prosser, C. F. 1992, AJ, 104, 645
Kurucz, R. L. 1979, ApJS, 40, 1

Lai, D. K., Smith, G. H., Bolte, M., et al. 2011, AJ, 141, 62

Lardo, C., Pancino, E., Mucciarelli, A., \& Milone, A. P. 2012, A\&A, 548, A107

Lee, J.-W., Carney, B. W., \& Balachandran, S. C. 2004, AJ, 128, 2388

Maccarone, T. J., \& Zurek, D. R. 2012, MNRAS, 423, 2

Marino, A. F., Milone, A. P., \& Lind, K. 2013, ApJ, 768, 27

Martell, S. L., Smith, G. H., \& Briley, M. M. 2008, AJ, 136, 2522

Meléndez, J., \& Cohen, J. G. 2009, ApJ, 699, 2017

Mészáros, Sz., Allende Prieto, C., Edvardsson, B., et al. 2012, AJ, 144, 120

Mészáros, Sz., Holtzman, J., García Pérez, A. E., et al. 2013, AJ, 146, 133

Milone, A. P., Bedin, L. R., Piotto, G., et al. 2008, ApJ, 673, 241

de Mink, S. E., Pols, O. R., Langer, N., \& Izzard, R. G. 2009, A\&A, 507, L1

Minniti, D., Peterson, R. C., Geisler, D., \& Claria, J. J. 1996, ApJ, 470, 953

O'Connell, J. E., Johnson, C. I., Pilachowski, C. A., \& Burks, G. 2011, PASP, 123,1139

Otsuki, K., Honda, S., Aoki, W., Kajino, T., \& Mathews, G. J. 2006, ApJL, 641, L117

Pinsonneault, M. H., Elsworth, Y., Epstein, C., et al. 2014, arXiv:1410.2503

Piotto, G., Bedin, L. R., Anderson, J., et al. 2007, ApJL, 661, L53

Ramírez, S. V., \& Cohen, J. G. 2002, AJ, 123, 3277

Pawa, S. V., \& Cohen, J. G. 2003, AJ, 125, 224

Roederer, I. U., \& Sneden, C. 2011, AJ, 142, 22

Shetrone, M. D. 1996, AJ, 112, 1517

Shetrone, M., Martell, S. L., Wilkerson, R., et al. 2010, AJ, 140, 1119

Smith, G. H., Shetrone, M. D., Bell, R. A., Churchill, C. W., \& Briley, M. M. 1996, AJ, 112, 1511

Smith, G. H., Shetrone, M. D., \& Strader, J. 2007, PASP, 119, 722

Smith, V. V., Cunha, K., Shetrone, M. D., et al. 2013, ApJ, 765, 16

Sneden, C. 1973, ApJ, 184, 839

Sneden, C., Kraft, R. P., Guhathakurta, P., Peterson, R. C., \& Fulbright, J. P. 2004, AJ, 127, 2162

Sneden, C., Kraft, R. P., Prosser, C. F., \& Langer, G. E. 1991, AJ, 102, 2001

Sneden, C., Kraft, R. P., Prosser, C. F., \& Langer, G. E. 1992, AJ, 104, 2121

Sneden, C., Kraft, R. P., Shetrone, M. D., et al. 1997, AJ, 114, 1964

Sneden, C., Pilachowski, C. A., \& Kraft, R. P. 2000, AJ, 120, 1351

Sobeck, J. S., Kraft, R. P., Sneden, C., et al. 2011, AJ, 141, 175

Steinhaus, H. 1956, Bull. Acad. Polon. Sci., 4, 801

Steinmetz, M., Zwitter, T., Siebert, A., et al. 2012, AJ, 132, 1645

Strutskie, M. F., et al. 2006, AJ, 131, 1163

Sweigart, A. V., \& Mengel, J. G. 1979, ApJ, 229, 624

Wilson, J., Hearty, F., Skrutskie, M. F., et al. 2012, SPIE, 8446, 84460H

Ventura, P., D’Antona, F., Mazzitelli, I., \& Gratton, R. 2001, ApJL, 550, L65

Ventura, P., di Criscienzo, M., Carini, R., \& D'Antona, F. 2013, MNRAS, 431,3642

Villanova, S., Geisler, D., \& Piotto, G. 2010, ApJL, 722, L18

Yong, D., Aoki, W., \& Lambert, D. L. 2006a, ApJ, 638, 1018

Yong, D., Aoki, W., \& Lambert, D. L. 2006b, ApJ, 639, 918

Yong, D., Grundahl, F., D’Antona, F., et al. 2009, ApJL, 685, L62

Yong, D., Grundahl, F., Nissen, P. E., Jensen, H. R., \& Lambert, D. L. 2005, A\&A, 438, 875

Yong, D., Karakas, A. I., Lambert, D. L., Chieffi, A., \& Limongi, M. 2008, ApJ, 689, 1031

Yong, D., Roederer, I. U., Grundahl, F., et al. 2014, MNRAS, 441, 3396

Zasowski, G., Johnson, J. A., Frinchaboy, P. M., et al. 2013, AJ, 146, 81 